\title{
Phase-Lag Heat Conduction in Multilayered Cellular Media with Imperfect Bonds
}

\author{
A. H. Akbarzadeh*, D. Pasini* \\ Department of Mechanical Engineering, McGill University, Montreal, QC, H3A 0C3, Canada
}

\begin{abstract}
We present a theoretical framework to study the thermal responses of onedimensional multilayered systems, functionally graded solid media, and porous materials. The method for thermal analysis resorts to non-Fourier heat conduction theories including three-phase-lag, dual-phase-lag, and hyperbolic heat conduction. The graded media are modeled as multilayered systems displaying finite numbers of layers. For each homogenous layer, the differential equations of heat conduction describing the wave-like three-phaselag are solved in closed-form in the Laplace domain. Solutions accounting for proper interfacial and boundary conditions are first presented to describe the thermal behavior of heterogeneous solids and porous media. Transient temperature and heat flux are obtained in time domain via fast Laplace inversion. We then apply the solutions obtained with each heat conduction theory to one-dimensional media and compare their thermal behavior. Finally, maps are presented to visualize the thermal responses of cellular materials, functionally graded cellular materials, and multilayered systems. For the latter, particular attention is devoted to investigate the impact of key attributes defining graded media, such as layer bond imperfections and material heterogeneity.
\end{abstract}

Keywords: Functionally graded material, Cellular solid, Porous multilayered composites, Heat conduction, Phase-lag theory, Thermal wave.

\section{Introduction}

The governing equations of heat conduction are generally described by the heat fluxtemperature gradient and the first-law of thermodynamics. If we use the conventional Fourier equation to solve the heat conduction problem, two problems emerge. First, the resulting differential equation, which is parabolic, turns out to predict an infinite thermal wave speed that is physically unrealistic. Second, for very low temperature, short-pulse thermal heating, as well as for micro-temporal and spatial scale applications, the results are not aligned with

\footnotetext{
*Corresponding authors: damiano.pasini@mcgill.ca and hamid.akbarzadeh@mcgill.ca, Department of Mechanical. Engineering, McGill University, Montreal, QC, H3A 0C3, Tel: +1(514) 398-6295, Fax: +1(514) 398-7365.
} 
experimental observations [1]. To reconcile these discrepancies, several non-Fourier heat conduction theories have been introduced. Cattaneo and Vernotte $(\mathrm{C}-\mathrm{V})$ first introduced a thermal relaxation time in the model with a hyperbolic heat conduction and finite thermal wave speed [2]. Although valuable, the $\mathrm{C}-\mathrm{V}$ model leads to results that cannot accurately describe experimental data, mainly because the microstructural effects in the heat transport process are overlooked. As a result, Tzou [3, 4] proposed a dual-phase-lag (DPL) model that could also account for microstructural interactions such as phonon-electron and phonon scattering via the phase-lag of temperature gradient, and fast transient effect of thermal waves through phase-lag of heat flux. A three-phase-lag (TPL) heat conduction theory was later proposed by Choudhuri [5] to encompass all previous theories for non-Fourier heat conduction. The fractional derivative of the phase-lag heat conduction was also introduced by Ezzat et al. [6, 7]. The use of phase-lags of heat flux, temperature gradient, and thermal displacement gradient in the TPL model is important to understand several phenomena, such as bioheat transfer in living tissues, exothermic catalytic reactions, and harmonic plane wave propagation.

The study of heat conduction with non-Fourier approaches has been the subject of several theoretical investigations [8-13]. These approaches attempt to predict phenomena that cannot be captured by classical Fourier theories of heat conduction. For example, Ramadan [14] presented a semi-analytic solution for heat conduction in a multilayered composite by using DPL theory. The TPL phase-field system for thermal flux was studied by Miranville and Quintanilla [15]. Wang et al. [16, 17] studied the non-Fourier heat conduction in carbon nanotubes based on the concept of thermomass. The transient temperature field of the DPL model around a partially insulated crack was studied by Hu and Chen [18]. Recently, Afrin et al. [19] employed the DPL model for heat conduction analysis in a gas-saturated porous medium subjected to a short-pulsed laser heating.

Other works in literature aim at studying the accuracy of thermal induced responses obtained with non-Fourier heat transport models. Babaei and Chen [20] investigated the generalized coupled thermopiezoelectric response of a functionally graded (FG) cylinder using the finite element method. The coupled and uncoupled transient thermopiezoelectric behavior of a one-dimensional (1D) FG rod was investigated by Akbarzadeh et al. [21, 22]. Hosseini zad et al. [23] used the classical and generalized coupled thermoelasticity to describe the behavior of thermoelastic waves at the interfaces of a layered medium. Banik and Kanoria [24] dealt with the TPL thermoelastic interactions in an FG unbounded medium subjected to periodically varying heat sources. Akbarzadeh et al. [25, 26] employed the higher-order shear deformation theory to study the classical coupled and uncoupled thermoelasticity of FG thick plates.

In automotive, naval, and aerospace applications, sudden temperature changes are commonly experienced by structural materials, such as multi-phase and fiber-reinforced composites [27]. An accurate thermal analysis, as studied in [28-35], is thus essential to predict the level of thermal-induced deformation in composite materials. Yang and Shi [36], for example, established a stability test for heat conduction in a 1D multilayered solid. For a multilayered hollow cylinder, Jain et al. [37] presented a closed-form expression containing a double-series for time-dependent asymmetric heat conduction. An exact solution for transient 
heat conduction in cylindrical multilayered composites was presented by Amiri Delouei et al. [38]. Akbarzadeh and Chen [39, 40] theoretically studied the effect of steady-state hygrothermal loading on the magnetoelectroelastic responses of homogeneous and heterogeneous media.

Laminated composites often contain imperfections, such as small voids and defects, at the interfaces where cracks initiate and propagate. For this reason, the multiphysics of imperfectly bonded composites has become a subject of study [41-45]. Duan and Krihaloo [46], for example, studied the effect of imperfect bonding between the inclusions and matrix on the effective thermal conductivity of heterogeneous media. Hatami-Marbini and Shodja [47] studied the stress field of multi-phase inhomogeneity systems with perfect/imperfect interfaces under uniform thermal and far-field mechanical loading.

The mismatch of thermal properties between bonded layers in laminated composites causes structural failure. This is one reason for which functionally graded materials (FGMs) with continuous transition of material constituents have been introduced. FGMs enable to greatly reduce thermal stresses and stress concentrations [48-50]. Since their appearance, FGMs have been the subject of intense research [51-59]. An approximate solution was developed by Ishigura et al. [60] and Tanigawa et al. [61] for transient Fourier heat conduction in an FGM plate by using piecewise homogeneous layers. A closed-form asymptotic solution was later obtained by Jin [62] for the short-time temperature field via a multilayered material model. Zhou et al. [63] derived exact solutions for the transient heat conduction in an FG strip in contact with a fluid. Using the conjugate gradient method, the inverse hyperbolic heat conduction problem in FG cylinders was solved by Yang et al. [64] to estimate the heat flux from the temperature measurements. A theoretical framework was also proposed by Wang [65] to analyze the transient thermal analysis in FG hollow cylinders via the state space approach as well as the initial parameter method.

All of the forementioned works dealt with solid media; nonetheless, porous materials, such as foams and lattices, can be effectively used for thermal management. Due to the increasingly growing number of applications, such as sandwich panels, heat exchangers, and heat shields [66, 67], several theoretical and experimental studies have been proposed to understand their thermal behavior [68-71]. Leong and Li [72] obtained the effective thermal conductivity of porous structures via a unit cell model. Sadeghi et al. [73] designed a test bed to measure thermal conductivity and thermal contact resistance of metallic foams under compressive loads. A three-dimensional finite element model for characterizing the elastic, dielectric, and piezoelectric properties of piezoelectric foam structures was recently developed by Challagulla and Venkatesh [74]. Furthermore, recent advances in manufacturing have enabled the engineering of FG foams, an element that further motivates research on their thermal behavior [75, 76]. For instance, Zhu et al. [77] considered the problem of minimizing the maximum temperature of a structure insulated by FG metallic foams under transient heat conduction.

Thermal analysis of heterogeneous solid and porous structures requires an accurate prediction of steady-state and transient temperature fields. This work theoretically investigates the non-Fourier heat conduction in graded solid media with application for the first time to cellular materials. It extends the DPL heat conduction analysis in a heterogeneous medium 
conducted by Akbarzadeh and Chen [78]. The paper provides a semi-analytic solution via Bessel functions and Laplace transform for 1D graded solid and porous media with perfect/imperfect bonding interfaces for TPL, DPL, and C-V heat conduction theories. The semi-analytic solutions obtained in this work enable modeling FG structures with an arbitrary material profile and predict their thermal properties. Closed-form solutions are obtained in terms of Bessel functions in Laplace domain in Section 3. Section 4 presents the thermal responses of 1D media obtained with alternative heat conduction theories. Finally, the effect of FGM profile, bonding imperfection, and heterogeneity is visualized into maps presented in Sections 5 and 6.

\section{Problem Definition and Governing Equations}

This section reports the governing equations of non-Fourier heat conduction in a onedimensional (1D) graded medium. Figure 1 shows its material gradients in a general 1D coordinate system $\vec{x}$, where the number of layers is $N$. The position of the inner and outer surfaces is $x_{i}$ and $x_{o} ; x_{n}(n=1,2, \ldots, N)$ is the inner surface of the $\mathrm{n}^{\text {th }}$ layer with $x_{1}=x_{i}$ and $x_{N+1}=x_{o}$. The heterogeneous medium is initially at ambient temperature $T_{0}$, and is here approximated by a piecewise profile consisting of $N$ homogenous layers .

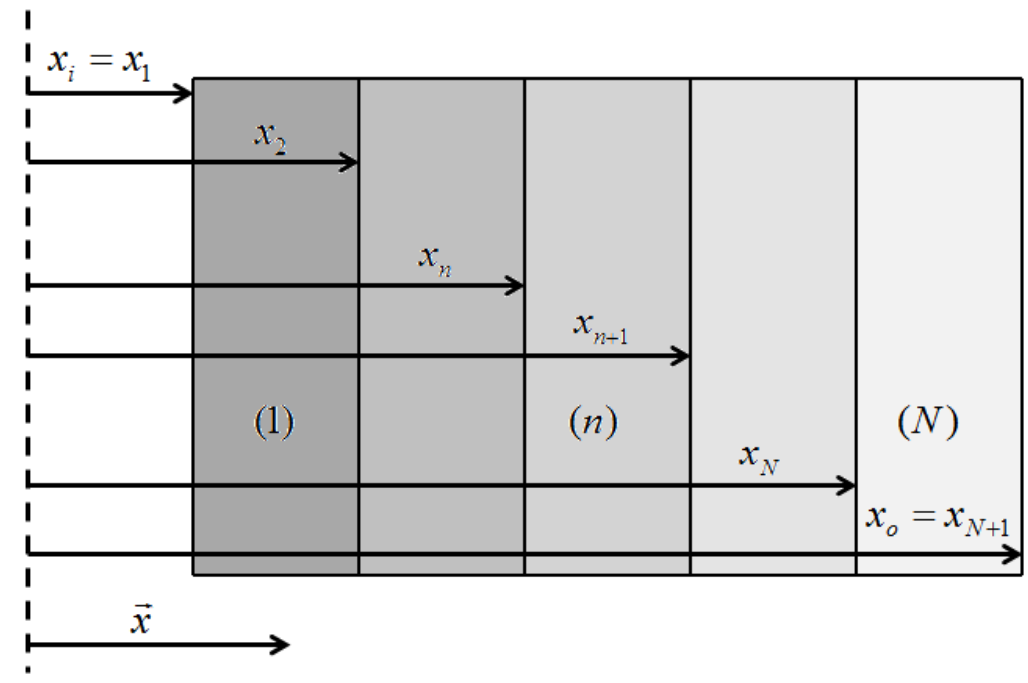

Fig. 1. Multilayered medium in a general 1D coordinate system

We can describe the general phase-lag heat conduction for each layer as [5, 79]:

$$
\vec{q}^{(n)}\left(\vec{x}, t+\tau_{q}^{(n)}\right)=-\left[K^{(n)} \vec{\nabla} T\left(\vec{x}, t+\tau_{T}^{(n)}\right)+K^{*(n)} \vec{\nabla} v\left(\vec{x}, t+\tau_{v}^{(n)}\right)\right]
$$

where $\vec{q}^{(n)}, T^{(n)}, v^{(n)}, K^{(n)}$, and $K^{*(n)}(n=1,2, \ldots, N)$ are, respectively, heat flux vector, absolute temperature, thermal displacement $\left(\dot{v}^{(n)}=T^{(n)}\right)$, thermal conductivity, and material constant characteristics of the TPL theory; $\tau_{q}^{(n)}, \tau_{T}^{(n)}$, and $\tau_{v}^{(n)}$ are also the phase-lag of heat 
flux, temperature gradient, and thermal displacement gradient, respectively. The position vector and gradient operator are $\vec{x}$ and $\vec{\nabla}$, while $t$ represents time. By using the second-order Taylor series expansion of equation (1) for $\tau_{q}^{(n)}$ and the first-order for $\tau_{T}^{(n)}$ and $\tau_{v}^{(n)}$, we obtain for $\dot{v}^{(n)}=T^{(n)}$ :

$$
\left(1+\tau_{q}^{(n)} \frac{\partial}{\partial t}+\frac{\tau_{q}^{(n)^{2}}}{2} \frac{\partial^{2}}{\partial t^{2}}\right) \vec{q}^{(n)}=-\left(\left(K^{(n)}+K^{*(n)} \tau_{v}^{(n)}\right) \vec{\nabla} T^{(n)}+K^{(n)} \tau_{T}^{(n)} \vec{\nabla} \dot{T}^{(n)}+K^{*(n)} \vec{\nabla} v^{(n)}\right)
$$

where overdot represents the time derivative. The equation of energy conservation can be written as:

$$
-\vec{\nabla} \cdot \vec{q}^{(n)}+R^{(n)}=\rho^{(n)} c_{p}^{(n)} \frac{\partial T}{\partial t}
$$

where $R^{(n)}, \rho^{(n)}$, and $c_{p}^{(n)}$ are: internal heat generation density, material density, and specific heat. By using Eqs. (2) and (3), we obtain the differential equation for phase-lag heat conduction in the general 1D coordinate system $\vec{x}$ :

$\left(1+\tau_{q}^{(n)} \frac{\partial}{\partial t}+\frac{\tau_{q}^{(n)^{2}}}{2} \frac{\partial^{2}}{\partial t^{2}}\left[\rho^{(n)} c_{p}^{(n)} \frac{\partial^{2} T^{(n)}}{\partial t^{2}}-\frac{\partial R^{(n)}}{\partial t}\right]=\left(\frac{\partial}{\partial x}+\frac{m}{x}\right)\left[\left(K^{(n)}+K^{*(n)} \tau_{v}^{(n)}\right) \frac{\partial^{2} T^{(n)}}{\partial x \partial t}+K^{(n)} \tau_{T}^{(n)} \frac{\partial^{3} T^{(n)}}{\partial x \partial t^{2}}+K^{*(n)} \frac{\partial T^{(n)}}{\partial x}\right]\right.$

where $m=0, m=1$, and $m=2$ refer respectively to 1D Cartesian, cylindrical, and spherical coordinate systems.

We note that Eq. (4) is obtained from the wave-like TPL model. Setting $K^{*(n)}=\tau_{v}^{(n)}=0$

leads to the wave-like DPL model. If besides this condition, we omit the term of $\tau_{q}^{(n)^{2}}$ of the second-order expansion, we obtain the diffusive-like DPL model. Similarly, the conditions $K^{*(n)}=\tau_{v}^{(n)}=\tau_{T}^{(n)}=0$ and $\tau_{q}^{(n)^{2}}=0$ leads to the $\mathrm{C}-\mathrm{V}$ model. Finally, disregarding $\tau_{q}^{(n)}$ in Eq. (4) generates the classical Fourier heat conduction. Readers interested in a detailed discussion on the characteristics of non-Fourier heat conduction theories are referred to $[5,79,80]$.

We also introduce the following dimensionless parameters for the heat conduction analysis in multilayered media:

$$
\begin{gathered}
\zeta=\frac{K^{\prime(1)} t}{x_{o}{ }^{2}}, \eta=\frac{x}{x_{o}}, \theta^{(n)}=\frac{T^{(n)}-T_{0}}{T_{0}}, Q_{x}^{(n)}=\frac{x_{o} q_{x}^{(n)}}{K^{(1)} T_{0}}, \varepsilon_{0}^{(n)}=\frac{K^{\prime(1)} \tau_{q}^{(n)}}{x_{o}{ }^{2}}, \delta_{0}^{(n)}=\frac{K^{\prime(1)} \tau_{T}^{(n)}}{x_{o}{ }^{2}} \\
\alpha_{0}^{(n)}=\frac{K^{\prime(1)} \tau_{v}^{(n)}}{x_{o}{ }^{2}}, K^{\prime(1)}=\frac{K^{(1)}}{\rho^{(1)} c_{p}^{(1)}}, K^{\prime(n)}=\frac{K^{(n)}}{\rho^{(n)} c_{p}^{(n)}}, \eta_{\gamma}=\frac{x_{i}}{x_{o}}, \theta_{w i}=\frac{T_{w i}-T_{0}}{T_{0}}, \theta_{w o}=\frac{T_{w o}-T_{0}}{T_{0}}
\end{gathered}
$$

where $T_{w i}$ and $T_{w o}$ are the temperature on the inner and outer surfaces.

\section{Methodology}

A general phase-lag model is here used to solve the transient and steady-state heat conduction in 1D multilayered media. We consider both perfectly and imperfectly bonded 
interfaces. The solutions can be applied to both porous materials and FGMs, as demonstrated in the following sections.

Using the dimensionless parameters (Eq. (5)) and in the absence of internal heat generation, the heat conduction equation (4) can be written as:

$$
\left(1+\varepsilon_{0}^{(n)} \frac{\partial}{\partial \zeta}+\frac{\varepsilon_{0}^{(n)^{2}}}{2} \frac{\partial^{2}}{\partial \zeta^{2}}\right) \frac{\partial^{2} \theta^{(n)}}{\partial \zeta^{2}}=\left[C_{T}^{(n)^{2}}+\left(\frac{K^{\prime(n)}}{K^{\prime(1)}}+C_{T}^{(n)^{2}} \alpha_{0}^{(n)}\right) \frac{\partial}{\partial \zeta}+\frac{K^{\prime(n)}}{K^{(1)}} \delta_{0}^{(n)} \frac{\partial^{2}}{\partial \zeta^{2}}\right]\left[\frac{m}{\eta} \frac{\partial \theta^{(n)}}{\partial \eta}+\frac{\partial^{2} \theta^{(n)}}{\partial \eta^{2}}\right]
$$

where $C_{T}^{(n)^{2}}=\frac{K^{*(n)} x_{0}{ }^{2}}{\rho^{(n)} c_{p}{ }^{(n)} K^{\prime(1)^{2}}}$. As explained in [1], the thermal wave speed in each layer for CV, wave-like DPL, and wave-like TPL models can be derived from Eq. (6) as:

$$
\begin{gathered}
C_{C-V}^{(n)}=\sqrt{\frac{K^{\prime(n)}}{K^{\prime(1)} \varepsilon_{0}^{(n)}}} \quad(\mathrm{C}-\mathrm{V}) \\
C_{D P L}^{(n)}=C_{T P L}^{(n)}=\frac{1}{\varepsilon_{0}^{(n)}} \sqrt{\frac{2 K^{\prime(n)} \delta_{0}^{(n)}}{K^{\prime(1)}}} \text { (Wave-like DPL and TPL) }
\end{gathered}
$$

From the above, we note that the dimensionless thermal wave speed in 1D media depends on the phase-lag of the heat flux, the phase-lag of temperature gradient, and the material properties of each layer of a multilayered composite, as well as $C_{D P L}^{(n)}=C_{T P L}^{(n)}=C_{C-V}^{(n)} \sqrt{\frac{2 \delta_{0}^{(n)}}{\varepsilon_{0}^{(n)}}}$. The difference of material properties between neighboring layers along with the interfacial imperfection cause the separation of the thermal wave into a transmitted and a reflected part $[14,23]$.

To solve the heat conduction equation (6) in time domain, the Laplace transform is here employed:

$$
\tilde{f}(s)=\int_{0}^{\infty} f(\zeta) \exp (-s \zeta) d \zeta
$$

where $\tilde{f}$ denotes the Laplace transform of function $f(\zeta)$ and $s$ is the Laplace variable. Applying Laplace transform to Eq. (6) and considering zero initial condition lead to:

$$
\eta^{2} \frac{\partial \tilde{\theta}^{(n)}}{\partial \eta^{2}}+m \eta \frac{\partial \tilde{\theta}^{(n)}}{\partial \eta}-D_{T P L}^{(n)} \eta^{2} \tilde{\theta}^{(n)}=0
$$

where

$$
D_{T P L}^{(n)}=\frac{\left(1+\varepsilon_{0}^{(n)} s+\frac{\varepsilon_{0}^{(n)^{2}}}{2} s^{2}\right) s^{2}}{C_{T}^{(n)^{2}}+\left(\frac{K^{\prime(n)}}{K^{(1)}}+C_{T}^{(n)^{2}} \alpha_{0}^{(n)^{2}}\right) s+\frac{K^{\prime(n)}}{K^{(1)}} \delta_{0}^{(n)} s^{2}}
$$

The differential equation (9) is solved in terms of Bessel functions as:

$$
\theta^{(n)}(\eta, s)=\eta^{\frac{1-m}{2}}\left(A_{1}^{(n)} J_{G^{(n)}}\left(I^{(n)} \eta\right)+A_{2}^{(n)} Y_{G^{(n)}}\left(I^{(n)} \eta\right)\right)
$$


where

$$
G^{(n)}=\left|\frac{m-1}{2}\right|, I^{(n)}=\sqrt{-D_{T P L}^{(n)}}
$$

and $A_{1}^{(n)}$ and $A_{2}^{(n)}(n=1,2, \ldots, N)$ are integration constants, and $J_{G^{(n)}}$ and $Y_{G^{(n)}}$ are $G^{(n)}$ th-order Bessel functions of the first and second kind. The heat flux is also written in Laplace domain using Eqs. (1), (5), (8), and (11) as follows:

$$
\tilde{Q}_{x}^{(n)}(\eta, s)=\frac{P_{T P L}^{(n)}}{2} \eta^{\frac{-m-1}{2}}\left(A_{1}^{(n)}\left(M^{(n)} J_{G^{(n)}}\left(I^{(n)} \eta\right)-2 I^{(n)} \eta J_{G^{(n)}+1}\left(I^{(n)} \eta\right)\right)+A_{2}^{(n)}\left(M^{(n)} Y_{G^{(n)}}\left(I^{(n)} \eta\right)-2 I^{(n)} \eta Y_{G^{(n)+1}}\left(I^{(n)} \eta\right)\right)\right)
$$

where

$$
M^{(n)}=2 G^{(n)}-(m-1), P_{T P L}^{(n)}=-\frac{C_{T}^{(n)^{2}}+\left(\frac{K^{(n)}}{K^{(1)}}+C_{T}^{(n)^{2}} \alpha_{0}^{(n)}\right) s+\frac{K^{(n)}}{K^{(1)}} \delta_{0}^{(n)} s^{2}}{s\left(1+\varepsilon_{0}^{(n)} s+\frac{\varepsilon_{0}^{(n)^{2}}}{2} s^{2}\right)}
$$

The integration constants in Eq. (11) are obtained by satisfying the following thermal boundary and interfacial conditions:

$$
\begin{gathered}
\left.\theta^{(1)}(\eta, \zeta)\right|_{\eta=\eta_{\gamma}}=\theta_{w i} f_{i}(\zeta) \\
\left.\frac{\chi_{T}^{(j)} K^{(1)}}{x_{o}} Q_{x}^{(j)}(\eta, \zeta)\right|_{\eta=\eta_{j+1}}=\left.\left(\theta^{(j)}(\eta, \zeta)-\theta^{(j+1)}(\eta, \zeta)\right)\right|_{\eta=\eta_{j+1}} \\
\left.Q_{x}^{(j)}(\eta, \zeta)\right|_{\eta=\eta_{j+1}}=\left.Q_{x}^{(j+1)}(\eta, \zeta)\right|_{\eta=\eta_{j+1}} \quad(j=1,2, \ldots, N-1) \\
\left.\theta^{(N)}(\eta, \zeta)\right|_{\eta=1}=\theta_{w o} f_{o}(\zeta) \quad
\end{gathered}
$$

where $\chi_{T}^{(j)}$ is the thermal compliance constant, or thermal contact resistance for the imperfect interface between layers $j$ and $j+1 ; f_{i}$ and $f_{o}$ are temporal functions for the applied thermal boundary conditions on the inner and outer surfaces. The bonding imperfection with thermally weak conduction has taken into account in Eq. (15) to obtain a reliable thermal analysis for multilayered composites [81-83]. The perfectly bonded interfaces could be also specified with $\chi_{T}^{(j)}=0$. In this analysis, a Heaviside step function, $f_{i}(\zeta)=f_{o}(\zeta)=H(\zeta)$, is used for the time-dependent functions. We note also that this procedure could model other transient thermal disturbances [78].

Using equations (11) and (13) along with thermal boundary and interfacial conditions (15) in Laplace domain results in the following algebraic equation that allows to obtain the integration constants:

$$
\left[K_{T P L}\right]_{2 N \times 2 N}\left\{X_{T P L}\right\}_{2 N \times 1}=\left\{F_{T P L}\right\}_{2 N \times 1}
$$

where $\left[K_{T P L}\right]$ is a $2 N \times 2 N$ matrix, $\left\{X_{T P L}\right\}$ is a $2 N \times 1$ vector of integration constants $\left\{X_{T P L}\right\}^{T}=\left\{\begin{array}{lllll}A_{1}^{(1)} & A_{2}^{(1)} & \ldots & A_{1}^{(N)} & A_{2}^{(N)}\end{array}\right\}$, and $\left\{F_{T P L}\right\}$ is a $2 N \times 1$ vector, whose components are given in Appendix A. Solving Eq. (16) gives the transient temperature change and heat flux 
in the Laplace domain. To restore temperature change and heat flux in the time domain, the fast Laplace inversion technique (FLIT) is employed. As mentioned in [72, 84, 85], the Laplace inversion of $\tilde{f}(\eta, s)$ at time $\zeta_{k}$ is obtained as follows:

$$
f\left(\eta, \zeta_{k}\right)=\frac{1}{2 \pi i} \int_{a-i \infty}^{a+i \infty} e^{s \zeta_{k}} \tilde{f}(\eta, s) d s \approx G(k)\left[-\frac{1}{2} \operatorname{Re}\{\tilde{f}(\eta, a)\}+\operatorname{Re}\left\{\sum_{p=0}^{N_{n}-1}(A(\eta, p)+i B(\eta, p)) W^{p k}\right\}\right]
$$

where

$$
\begin{gathered}
A(\eta, p)=\sum_{q=0}^{L_{n}} \operatorname{Re}\left(\tilde{f}\left(\eta, a+i\left(p+q N_{n}\right) \frac{2 \pi}{T_{\text {total }}}\right), B(\eta, p)=\sum_{q=0}^{L_{n}} \operatorname{Im}\left(\tilde{f}\left(\eta, a+i\left(p+q N_{n}\right) \frac{2 \pi}{T_{\text {total }}}\right)\right.\right. \\
G(k)=\frac{2}{\zeta_{\text {total }}} e^{a k \Delta \zeta}, \Delta \zeta=\frac{\zeta_{\text {total }}}{N_{n}}, W=e^{i \frac{2 \pi}{N_{n}}}, i=\sqrt{-1}
\end{gathered}
$$

where $\zeta_{\text {Total }}$ is the total dimensionless time interval for the Laplace inversion, and $a$ is an arbitrary real number larger than all the real parts of the singularities present in the function $\tilde{f}(\eta, s) ; L_{n}$ and $N_{n}$ are accuracy parameters of FLIT defined in the Laplace inversion by Durbin [84].

The following steady-state solutions for a thermally induced 1D medium can be also obtained by using the steady-state form of Eqs. (1) and (3) as well as Eq. (5):

$$
\begin{gathered}
\theta_{s}^{(n)}=A_{1 s}^{(n)}+A_{2 s}^{(n)} \eta^{1-m}, Q_{x s}^{(n)}=-(1-m) \frac{K^{(n)}}{K^{(1)}} A_{2 s}^{(n)} \eta^{-m} \\
\theta_{s}^{(n)}=A_{1 s}^{(n)}+A_{2 s}^{(n)} \ln (\eta), Q_{x s}^{(n)}=-\frac{K^{(n)}}{K^{(1)}} \frac{A_{2 s}^{(n)}}{\eta}
\end{gathered}
$$

(for $m=1)(19 \mathrm{a})$

where $\theta_{s}^{(n)}$ and $Q_{x s}^{(n)}(n=1,2, \ldots, N)$ represent the steady-state temperature change and heat flux for each layer of the multilayered composite. Satisfying the steady-state form of boundary and interfacial conditions (15) leads to the following algebraic equation to obtain the integration constants $A_{1 s}^{(n)}$ and $A_{2 s}^{(n)}$ :

$$
\left[K_{s}\right]_{2 N \times 2 N}\left\{X_{s}\right\}_{2 N \times 1}=\left\{F_{s}\right\}_{2 N \times 1}
$$

where $\left[K_{s}\right]$ is a $2 N \times 2 N$ matrix, $\left\{X_{s}\right\}$ is a $2 N \times 1$ vector of integration constants $\left\{X_{s}\right\}^{T}=\left\{\begin{array}{lllll}A_{1 s}^{(1)} & A_{2 s}^{(1)} & \ldots & A_{1 s}^{(N)} & A_{2 s}^{(N)}\end{array}\right\}$, and $\left\{F_{s}\right\}$ is a $2 N \times 1$ vector whose components are specified in Appendix B. It is worth mentioning that there is no difference between the steady-state solutions of non-Fourier and classical Fourier heat conduction models, as seen in Eq. (19).

The methodology developed above is initially used in the next section to compare and discuss the thermal responses of the heat conduction theories under investigation. The method is then applied to FG and multilayered media in the following sections. 


\section{Comparison of TPL, DPL, and C-V Non-Fourier Heat Conduction Theories}

Figure 2 shows the thermal responses of a homogeneous sphere $(m=2)$, with inner and outer radius $x_{i}=0.6$ and $x_{o}=1$, subjected to a sudden temperature rise. The thermal responses obtained with TPL, DPL, and C-V models are plotted. The curves are obtained by assuming an initial ambient temperature of $T_{0}=300 \mathrm{~K}$ for the medium. In addition, its inner surface is at ambient temperature $\left(T_{w i}=300 \mathrm{~K}\right)$, whereas the outer surface experiences a sudden Heaviside temperature rise $T_{w o}=600 \mathrm{~K}$. The dimensionless phase lags of heat flux, temperature gradient, and thermal displacement gradient are respectively assumed $\varepsilon_{0}=0.35$, $\delta_{0}=0.25$, and $\alpha_{0}=0.15$, as those in [6,13, and 14]. The TPL parameter is assumed as $C_{T}{ }^{2}=2$. We consider materials common in the electronic industry and for thermal insulation [86], with thermal properties given in Table 1. It is worth to note that the value of the thermal relaxation time or the phase-lag of heat flux $\left(\tau_{q}\right)$ for non-Fourier heat conduction theories varies between $10^{-14} s$ and $10^{3} s$ for metals, organic tissues, and materials with microstructural non-homogeneity, such as polyethylene/graphite nanosheets. We observe that the phase-lag of the temperature gradient $\left(\tau_{T}\right)$ follows also similar variations, although in literature, no exact value for the phase-lag of the thermal displacement gradient $\left(\tau_{v}\right)$ exists [3, 87-90].

Table 1 Thermal properties

\begin{tabular}{cccc}
\hline \multicolumn{4}{c}{ Table 1 Thermal properties } \\
\hline \\
\hline \hline$\left(\frac{W}{m K}\right)$ & $\rho\left(\frac{K g}{m^{3}}\right)$ & $c_{p}\left(\frac{J}{K g K}\right)$ \\
\hline$C u$ & 386 & 8960 & 386 \\
$A g$ & 419 & 10490 & 233 \\
$A u$ & 315 & 19300 & 126 \\
\hline \hline
\end{tabular}

The sudden temperature rise on the outer surface of the sphere causes the thermal wave to propagate towards the inner surface. The temperature distribution through the wall thickness of the sphere at the dimensionless time $\zeta=0.098$ and the temperature time-history at the middle surface of sphere $\eta=0.8$ are illustrated, respectively, in Figs. 2a and 2b. The hyperbolic TPL, hyperbolic DPL, and C-V models are used to plot the curves. The curve of the C-V heat conduction theory reproduces that obtained in [13], while that of the hyperbolic DPL model the one in [78]. We note that the results for a homogenous sphere are independent of the material properties except the phase-lags. 


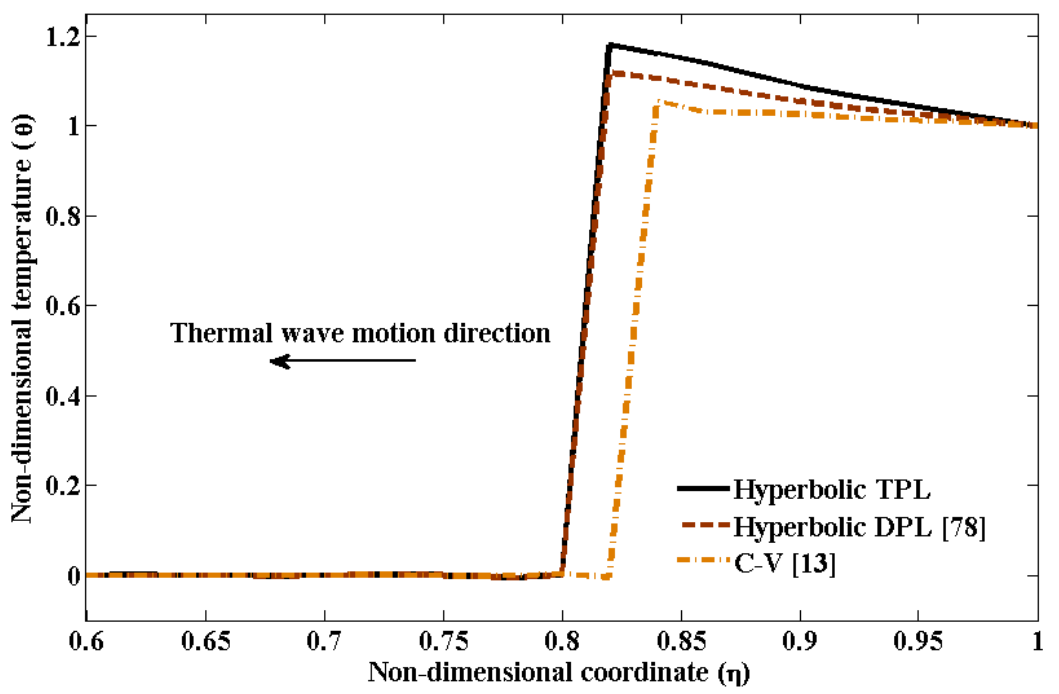

(a)

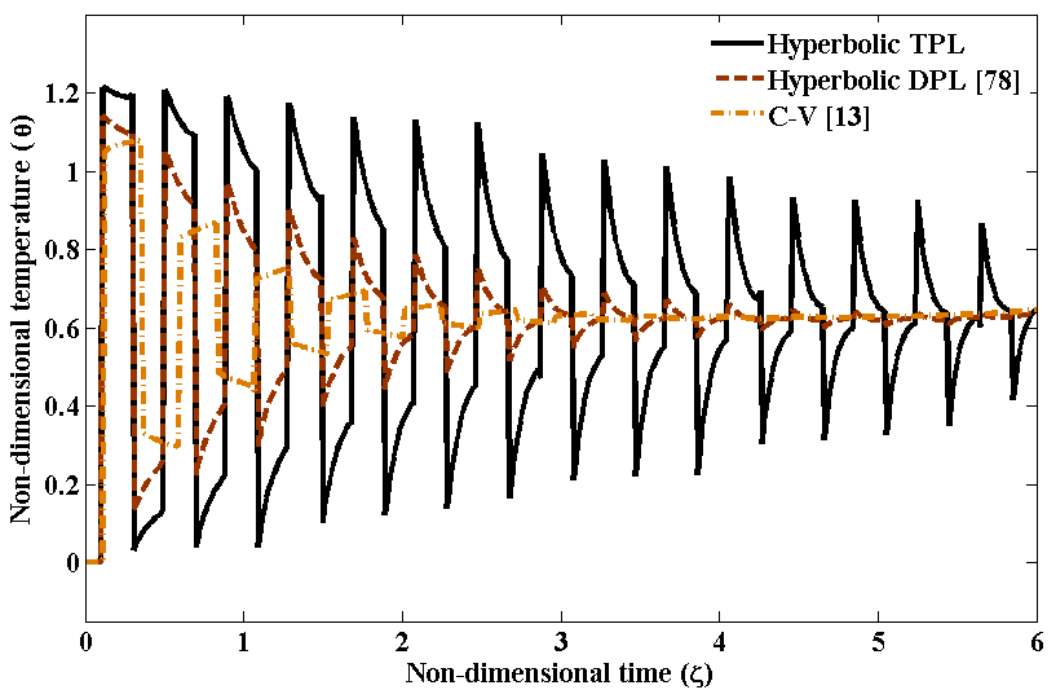

(b)

Fig. 2. (a) Temperature distribution and (b) temperature time-history obtained with TPL, DPL and C-V for a homogenous sphere $\left(m=2, \eta=0.8, \varepsilon_{0}=0.35, \delta_{0}=0.25, \alpha_{0}=0.15, C_{T}{ }^{2}=2\right)$

Fig. 2a shows that hyperbolic-type heat conduction theories can predict the thermal wave propagation with a finite thermal wave speed through the medium, as a result of the hyperbolic nature of the governing equations. Nonetheless, the thermal wave characteristics, such as thermal wave speed and temperature difference at the thermal wave front, differ. For Fourier and parabolic-type heat conduction theories with diffusive-like thermal behavior, one can refer to references $[78,79,90]$. On the other hand, the thermal wave for the hyperbolic DPL and TPL models is faster than that obtained with the C-V model, an observation aligned 
with the premise of Eq. (7). Furthermore, Fig. 2a shows the thermal wave speed for TPL and DPL is $12 \%$ higher than that for C-V. While the thermal waves predicted with TPL and DPL show to move at a given wave speed, the temperature jump on the thermal wave front of former is around 5\% larger than that of the latter.

Fig. $2 b$ illustrates that the temperature history in the middle of the plate oscillates around the steady-state temperature. The maximum transient temperature of the TPL is $12 \%$ higher than that of the $\mathrm{C}-\mathrm{V}$ model, while the maximum transient temperature of the DPL is $6 \%$ higher than for $\mathrm{C}-\mathrm{V}$ model. Since non-Fourier approaches seem to better approximate experimental results $[91,92]$, we apply them to graded media subjected to thermal shocks in section 5 .

\section{Application of TPL and DPL Theories to FG Media}

The methodology explained in section 3 is here applied to model FG solid and porous media. Before presenting their thermal responses, we start introducing the homogenized properties of porous media and the piecewise profile approximation for FGMs.

\subsection{Cellular Materials}

Herein, the focus is on cellular materials, including foams and lattices [93]. Since a fully detailed microscale analysis of porous media is computationally expensive, a multiscale model based on homogenization theory is used. We consider a representative volume element of the cellular materials, obtain its properties, and use them as the effective material attributes of its homogenous solid counterpart [94-97].

Following this approach, the effective specific heat of foams can be obtained by the classical rule of mixture which includes the contribution of the solid and gas as [67]:

$$
\bar{\rho} \bar{c}_{p}=\left(\rho_{s} c_{p_{s}}\right) \rho_{r}+\left(\rho_{g} c_{p_{g}}\right)\left(1-\rho_{r}\right)
$$

where subscripts " $s$ " and " $g$ " represent the material properties of the solid and gas, and overbar parameters specify the effective material properties; $\rho_{r}$ is the relative density defined as: $\rho_{r}=\frac{\bar{\rho}}{\rho_{s}}$. If we neglect the heat convection of the gas due to the small size of pores, we can approximate its thermal conductivity as [67]:

$$
\bar{K}=\frac{1}{3}\left(\rho_{r}+2 \rho_{r}^{\frac{3}{2}}\right) K_{s}+\left(1-\rho_{r}\right) K_{g}
$$

In this work, we assume the specific heat and thermal conductivity of the gas to be equal to those of dry air: $\rho_{g} c_{p_{g}}=1.006 \times 10^{3} \frac{\mathrm{J}}{\mathrm{KgK}}$ and $K_{g}=0.025 \frac{\mathrm{W}}{\mathrm{mK}}$. Equations (21) and (22) are thus the effective thermal properties that can be used to analyze the heat conduction in multilayered composites with porous layers.

\subsection{Functionally Graded Cellular Materials}


Functionally graded structures are modelled here as a perfectly bonded multilayered composite, where the material properties of subsequent homogenous layers vary according to the FGM profile [60-62, 94]. The property profile (Fig. 3) is described by a piecewise function of homogenous layers expressed as a function of the volume fraction $\mathrm{V}$. The accuracy of the approximated property profile and predicted thermal responses of FGMs increases with the number of artificial layers, $N$, in thermal solutions provided in Section 3. In this paper, the FGM profiles are modelled with the following power-laws (P-FGM) [98102]:

$$
\chi=\chi_{i}+\left(\chi_{o}-\chi_{i}\right)\left(\frac{\eta-\eta_{\gamma}}{1-\eta_{\gamma}}\right)^{n_{\chi}}
$$

, Sigmoid (S-FGM):

$$
\begin{gathered}
\chi=\chi_{i}+\frac{\left(\chi_{o}-\chi_{i}\right)}{2}\left(\frac{2\left(\eta-\eta_{\gamma}\right)}{1-\eta_{\gamma}}\right)^{n_{\chi}} \quad\left(\eta_{\gamma} \leq \eta \leq \frac{1+\eta_{\gamma}}{2}\right) \\
\chi=\chi_{i}+\left(\chi_{o}-\chi_{i}\right)\left(1-\frac{1}{2}\left(\frac{2(\eta-1)}{\eta_{\gamma}-1}\right)^{n_{\chi}}\right) \quad\left(\frac{1+\eta_{\gamma}}{2} \leq \eta \leq 1\right)
\end{gathered}
$$

, and Exponential (E-FGM) forms:

$$
\chi=\chi_{o} \exp \left(\ln \left(\frac{\chi_{i}}{\chi_{o}}\right)\left(\frac{1-\eta}{1-\eta_{\lambda}}\right)^{n_{\chi}}\right)
$$

where $\chi$ can be any material property of FGMs and $n_{\chi}$ is the corresponding nonhomogeneity index. This straightforward approximation of FGMs could be used for functionally graded solids (FGS) as well as functionally graded cellular (FGC) materials. 

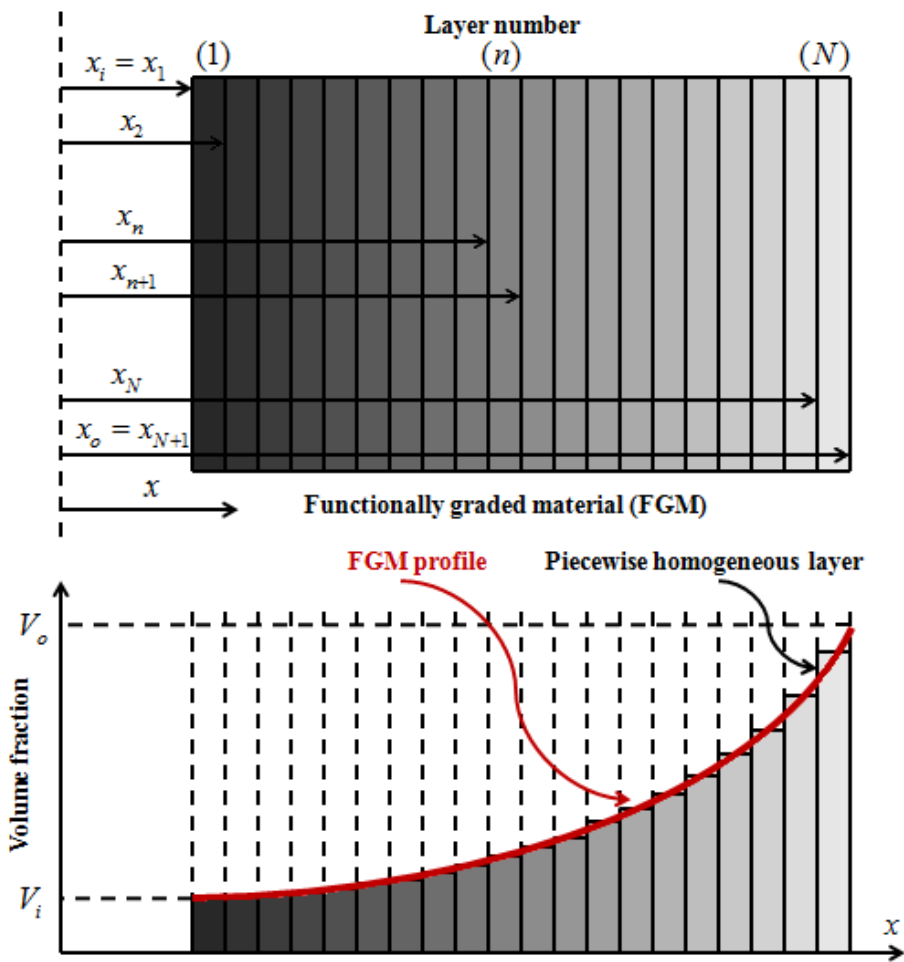

Fig. 3 The piecewise homogenous layers for modeling of FGMs

\subsection{Thermal Responses of FGC Media}

Functionally graded cellular materials (FGCMs) are efficient lightweight alternatives to multilayered composites, which often suffer from bonding imperfections causing, for example, undesired separation of thermal waves. FGCMs, on the other hand, offer continuous transition of material properties that govern the medium response via their FGM profile.

To study the thermal responses of FGC media, we examine a practical example: an FGC cylinder with inner and outer radius $x_{i}=0.6$ and $x_{o}=1$ and relative density of inner and outer layers $\rho_{r_{i}}=0.1$ and $\rho_{r_{o}}=1$. This cylinder is subjected to a sudden temperature rise $T_{w o}=600 \mathrm{~K}$. The continuous transition of relative density throughout the FGC cylinder is shown in Figure 4 for P-FGM, S-FGM, and E-FGM profiles and non-homogeneity index $n_{\rho}=2$. 


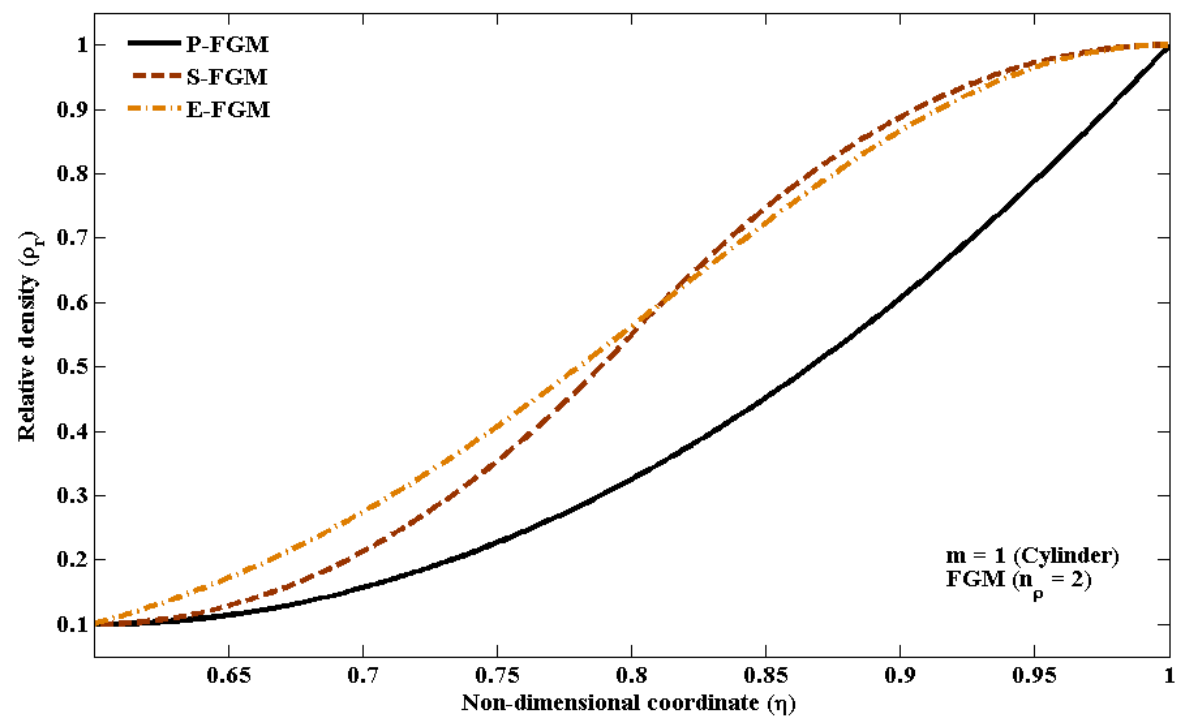

Fig. 4 Effect of FGM profile on the relative density distribution of an FGC cylinder $\left(m=1, F G M\left(n_{\rho}=2\right)\right)$

Through Eqs. (21) and (22) we can gather that the variation of relative density controls the effective thermal properties of the FGC cylinder. This is visualized in Figures 5 and 6, where the transient and steady-state temperature and heat flux distributions are plotted for the hyperbolic DPL model. Since the changes of relative density of the FG cylinder with $n_{\rho}=2$ in S-FGM and E-FGM profile are greater than the P-FGM, the thermal wave speed in the SFGM and E-FGM cylinders are also greater than that of the P-FGM cylinder.

Fig. 7 shows the temperature time-history of the middle of the cylinder roughly resemble for all FGM profiles; nonetheless, the absolute value of steady-state heat flux in E-FGM is higher than that obtained with S-FGM and P-FGM. From this observation, we gather the importance of selecting an appropriate FGM profile so as to satisfy the design requirements of FGC media subjected to thermal shocks. Furthermore, Figs. 5a and 6a show that an increase in the calculations of the spatial points in each layer creates numerical noises before and after the wave front, a phenomenon previously observed [23]. The nature of abrupt Heaviside thermal excitations could be mentioned as one culprit. 


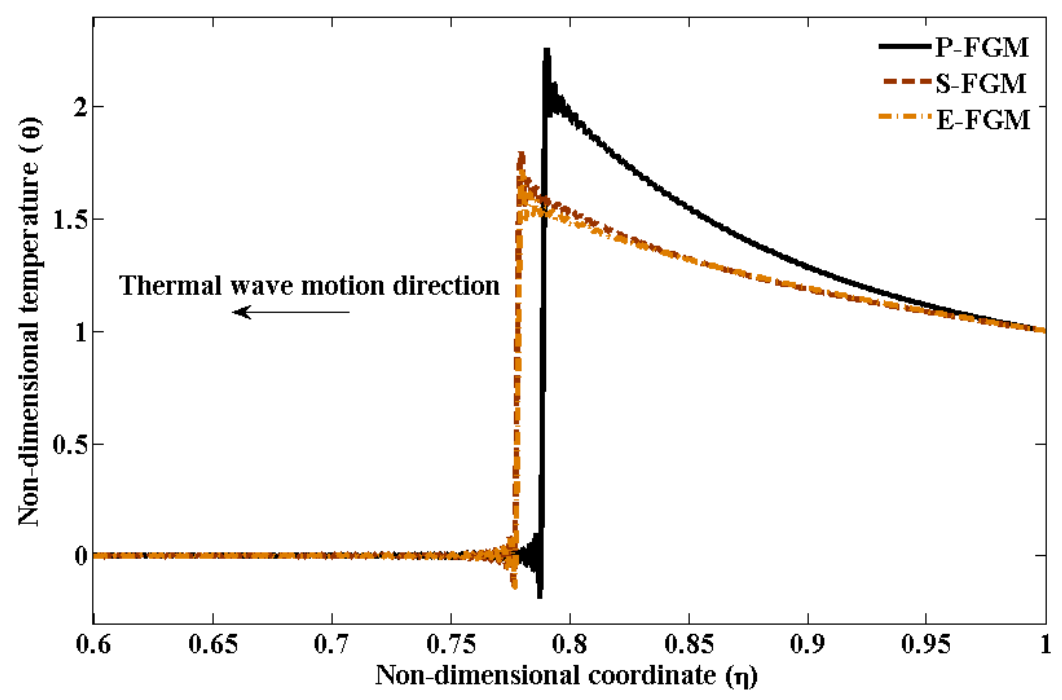

(a)

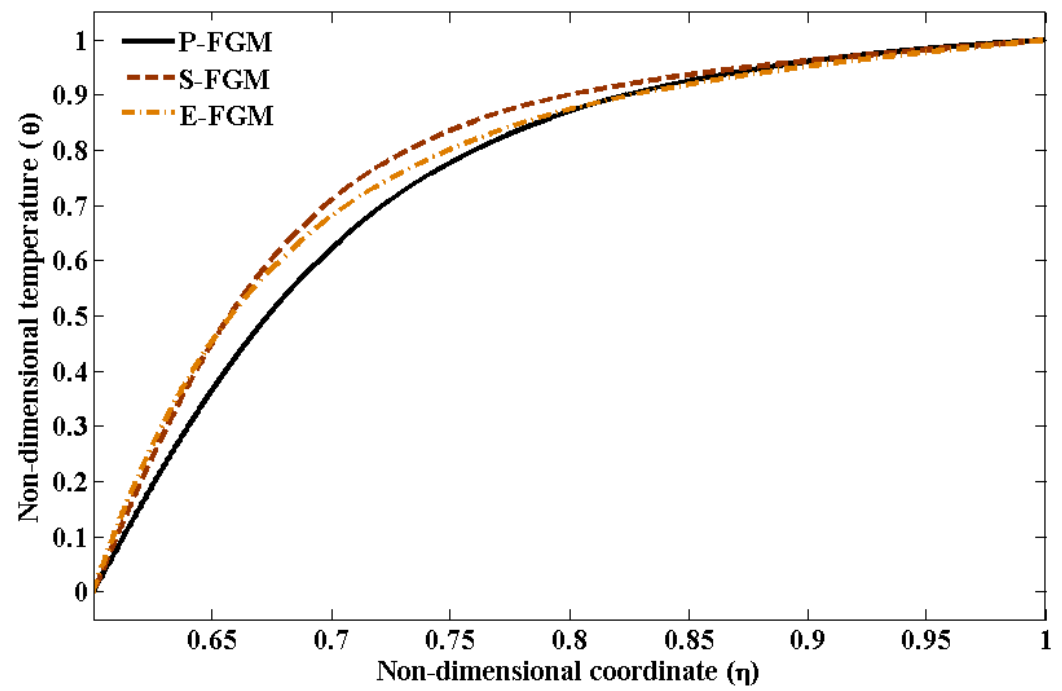

(b)

Fig. 5 Effect of FGM profile of an FGC cylinder on temperature distribution at (a) dimensionless time $\zeta=0.084$ and (b) steady-state $\left(m=1, \varepsilon_{0}=0.35, \delta_{0}=0.25, \operatorname{FGM}\left(\mathrm{n}_{\rho}=2\right)\right.$, Hyperbolic DPL) 


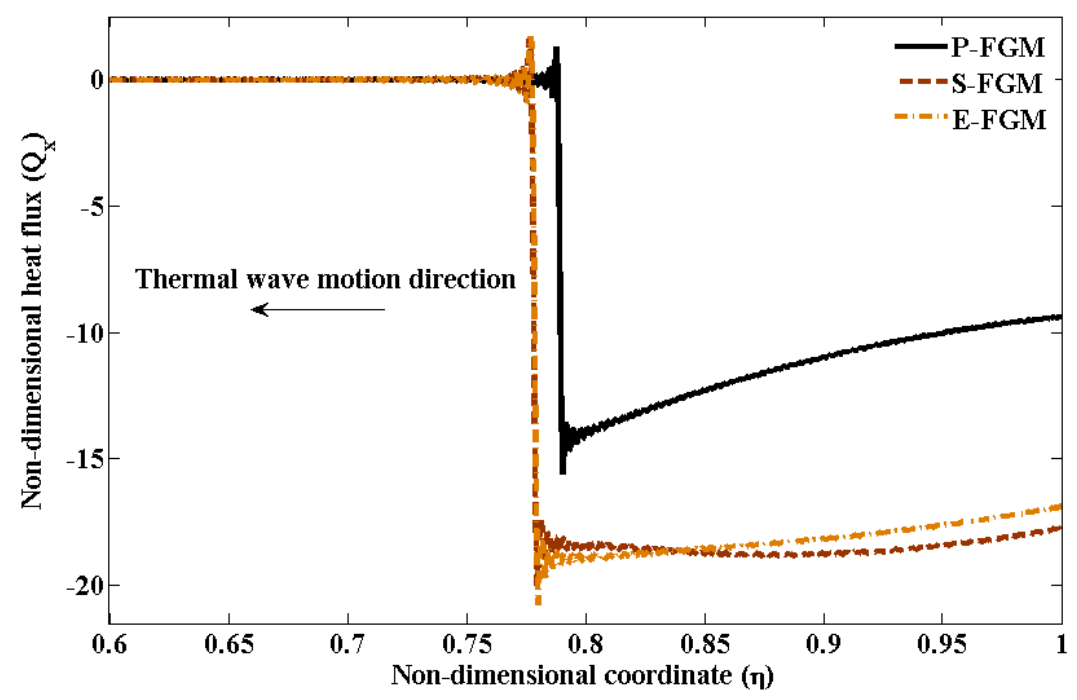

(a)

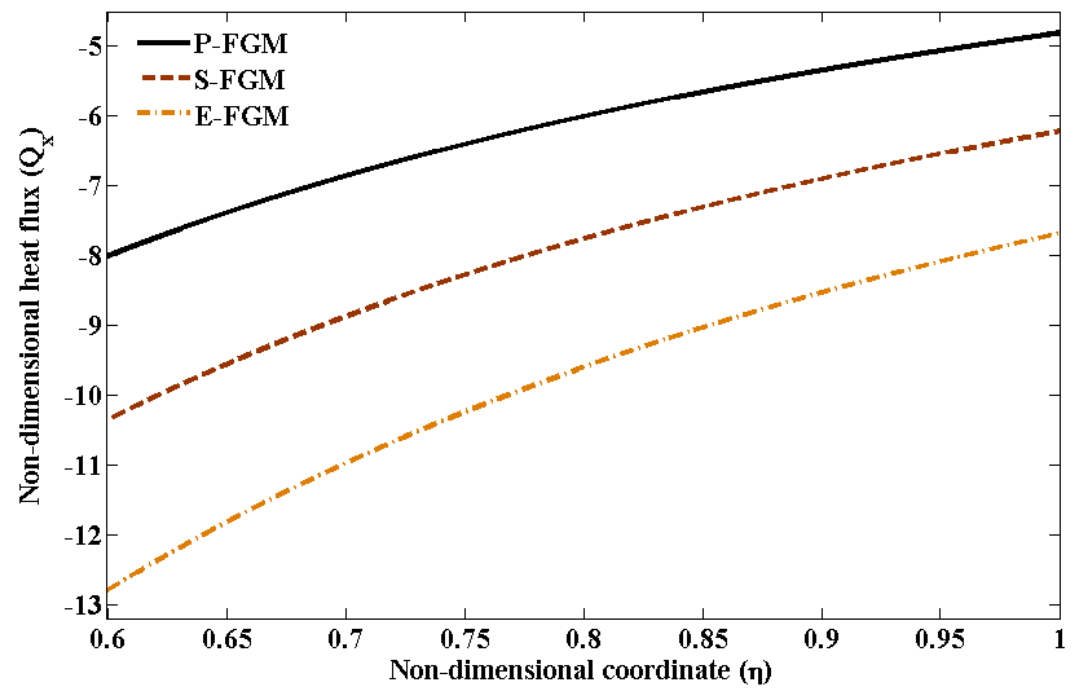

(b)

Fig. 6 Effect of FGM profile of an FGC cylinder on heat flux distribution at (a) dimensionless time $\zeta=0.084$ and (b) steady-state $\left(m=1, \varepsilon_{0}=0.35, \delta_{0}=0.25, \operatorname{FGM}\left(\mathrm{n}_{\rho}=2\right)\right.$, Hyperbolic DPL $)$ 


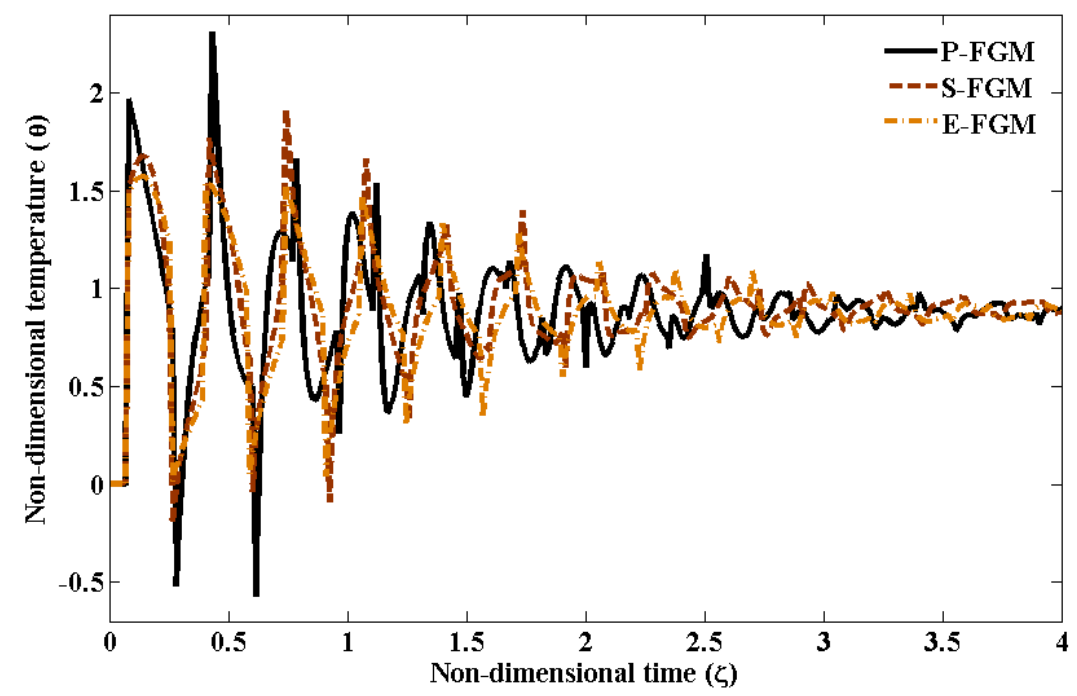

(a)

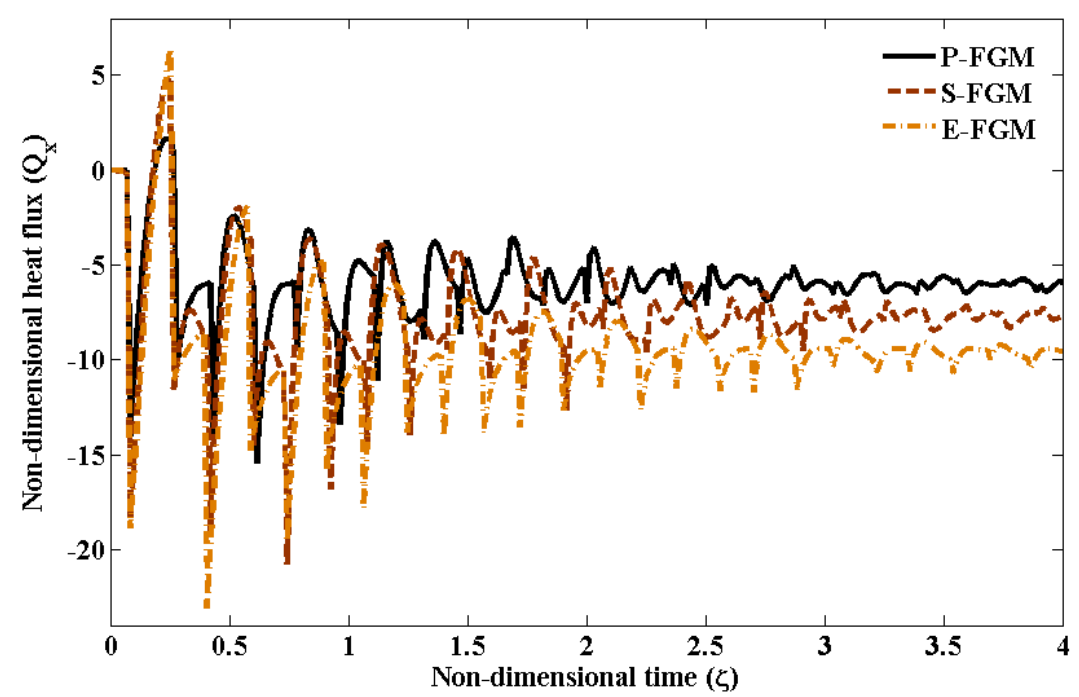

(b)

Fig. 7 Effect of FGM profile of an FGC cylinder on (a) temperature time-history and (b) heat flux timehistory at the middle of $\mathrm{FG}$ porous cylinder

$\left(m=1, \varepsilon_{0}=0.35, \delta_{0}=0.25, \operatorname{FGM}\left(\mathrm{n}_{\rho}=2\right)\right.$, Hyperbolic DPL $)$

\section{Application of TPL and DPL Theories to Multilayered Media}

This section studies the thermal behavior of solid and porous multilayered media as well as the influence of bonding imperfection, the heterogeneity of bonded layers, and the relative density of a porous core in multilayered media. 


\subsection{Influence of Bonding Imperfection}

Figure 8 illustrates the effect of imperfectly bonded interfaces on the temperature and heat flux distribution of a bilayered cylinder $(m=1)$ with inner radius $x_{i}=0.6$ and outer radius $x_{o}=1$. The cylinder is subjected to a sudden temperature rise of $T_{w o}=600 \mathrm{~K}$, and the hyperbolic DPL model is used for heat conduction prediction.

To study the effect of dimensionless thermal compliance constant due to bonding imperfection $\chi_{T}$, we consider a bilayered cylinder with two bonded $C u$ layers. This case reduces to that examined in [78] for perfectly bonded interfaces $\left(\chi_{T}=0\right)$, which thus represents here a baseline for comparison.

Figures $8 \mathrm{a}$ and $8 \mathrm{~b}$ plot temperature and heat flux for the dimensionless time $\zeta=0.126$. They illustrate that the thermal excitation causes the thermal wave to propagate towards the inner surface of the cylinder. The bonding imperfection causes the separation of the initial thermal wave into a transmitted and a reflected part, each travelling, at a given thermal wave speed of $C_{D P L}$, towards the inner and outer surfaces of cylinder respectively. Although the temperature is discontinuous at the layer interface of the cylinder, the radial heat flux is continuous at the interface; these observations are consistent with the thermal conditions expressed in Eq. (15).

Figure 9 shows that the absolute value of the transient and steady-state temperature and heat flux in the middle of the inner layer $(\eta=0.7)$ decreases by stiffening the thermal compliance of the interfaces. In addition, Figs. 8 and 9 show that the thermal compliance amplifies the temperature difference at the interface of cylinder and shrinks the heat flux transmitted through the interface. As a result of the thermally disturbed outer layer, a thermal insulation is developed for the inner layer.

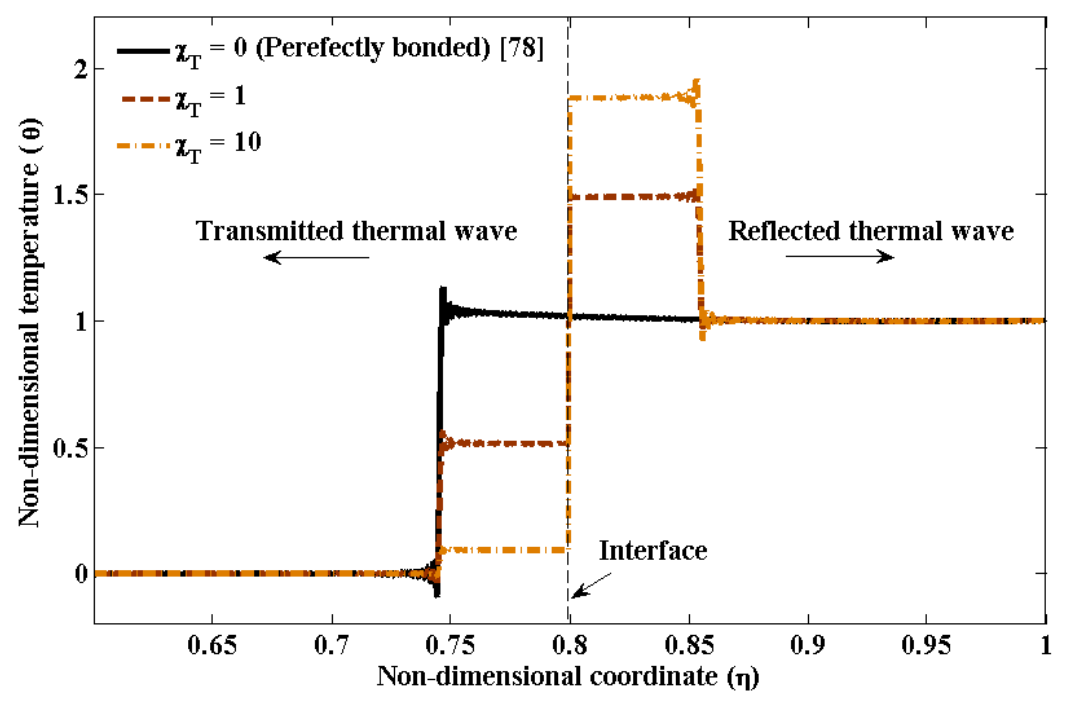

(a) 


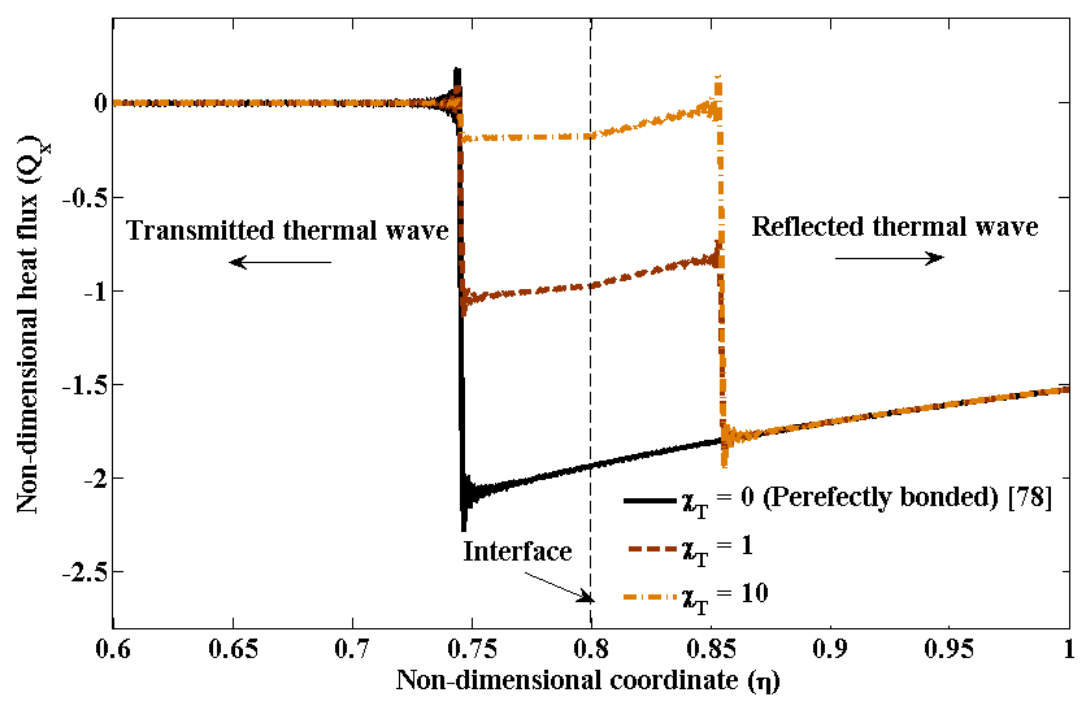

(b)

Fig. 8 Effect of thermal compliance on the distribution of (a) temperature and (b) heat-flux at dimensionless time $\zeta=0.126\left(m=1, \varepsilon_{0}=0.35, \delta_{0}=0.25\right.$, Hyperbolic DPL)

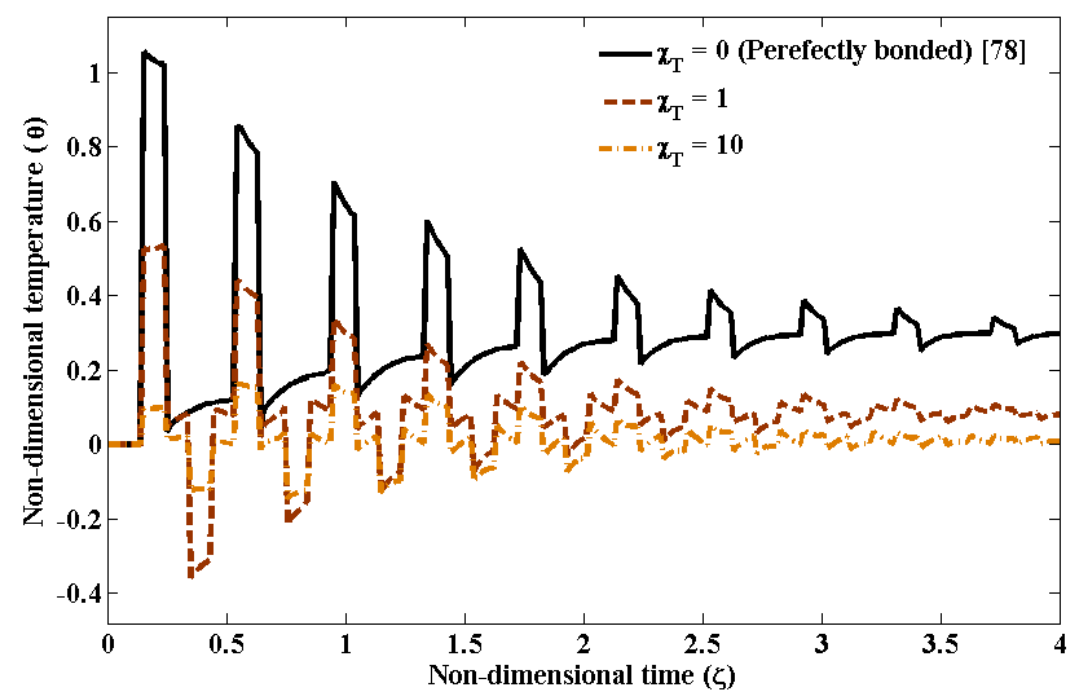

(a) 


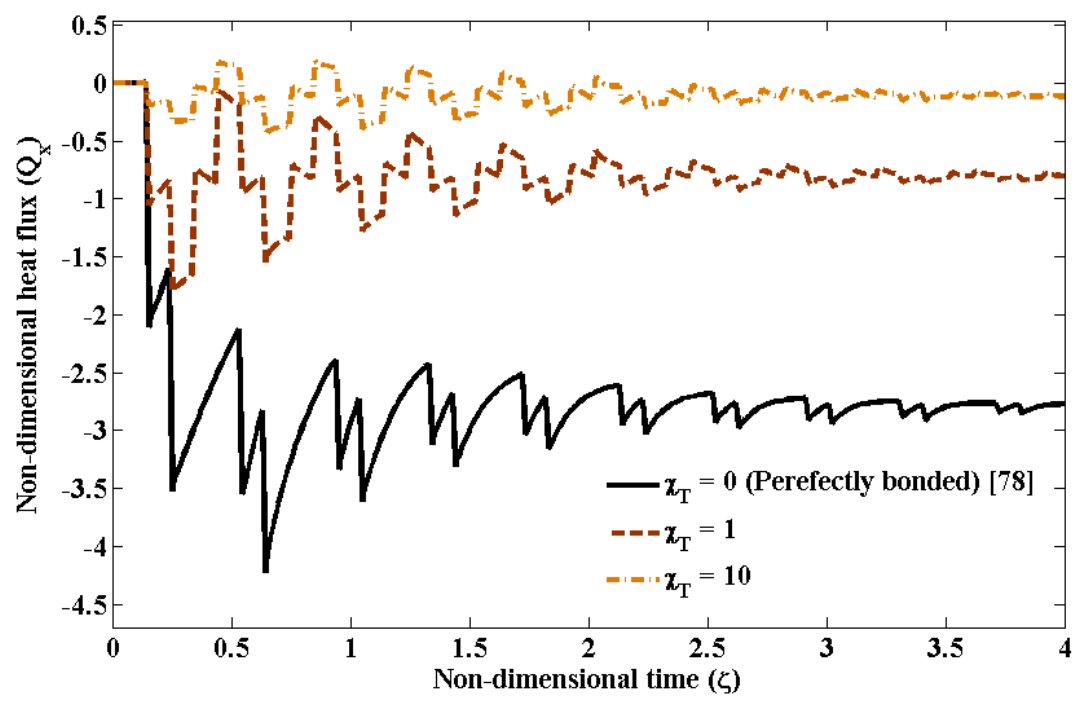

(b)

Fig. 9 Effect of thermal compliance on: (a) temperature and (b) heat flux time-histories at $\eta=0.7$

$$
\left(m=1, \varepsilon_{0}=0.35, \delta_{0}=0.25\right. \text {, Hyperbolic DPL) }
$$

\subsection{Influence of Heterogeneity}

Bonding imperfections often trigger thermal wave separation in a multilayered system. Material heterogeneity is generally the main culprit. Figure 10 shows the influence of heterogeneity of the middle layer in a sandwich slab $(m=0)$. By using the hyperbolic TPL model, the position of the inner and outer surfaces are assumed to be $x_{i}=0.7$ and $x_{o}=1$. The perfectly bonded sandwich slab $\left(\chi_{T}=0\right)$ has inner and outer layers of $C u$ while the middle layer of the slab consists of either $C u$ or $A g$ or $A u$. For the layer arrangement $C u / C u / C u$, $\mathrm{Cu} / \mathrm{Ag} / \mathrm{Cu}$, and $\mathrm{Cu} / \mathrm{Au} / \mathrm{Cu}$, we observe that the thermal waves move towards the inner layer of the slab. On the other hand, thermal wave separation occurs for the heterogeneous material arrangements $\mathrm{Cu} / \mathrm{Ag} / \mathrm{Cu}$, and $\mathrm{Cu} / \mathrm{Au} / \mathrm{Cu}$. This is caused by the meeting of a thermal wave front at an interface between layers with dissimilar material properties, thereby generating waves with reflected and transmitted portions. Figs. 10a and 10b depict this phenomenon at different dimensionless timeframe. Figure 10a shows that the thermal wave speed at the middle layer of $A g$ is higher than that of $A u$ due to their thermal properties; nonetheless, the transient temperature at the middle layer of $A g$ is lower than $A u$. We note here that the thermal wave transmits and reflects from the interfaces; it also reflects back from the inner and outer surfaces of the slab at $x_{i}=0$ and $x_{o}=1$ to finally reach the steady-state temperature. 


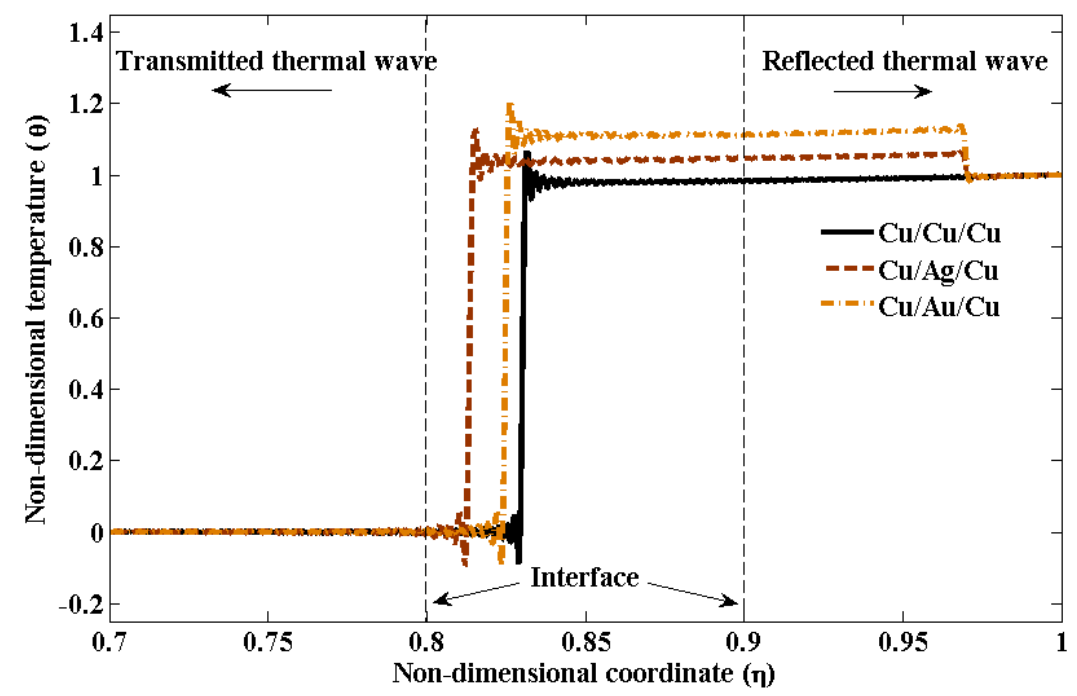

(a)

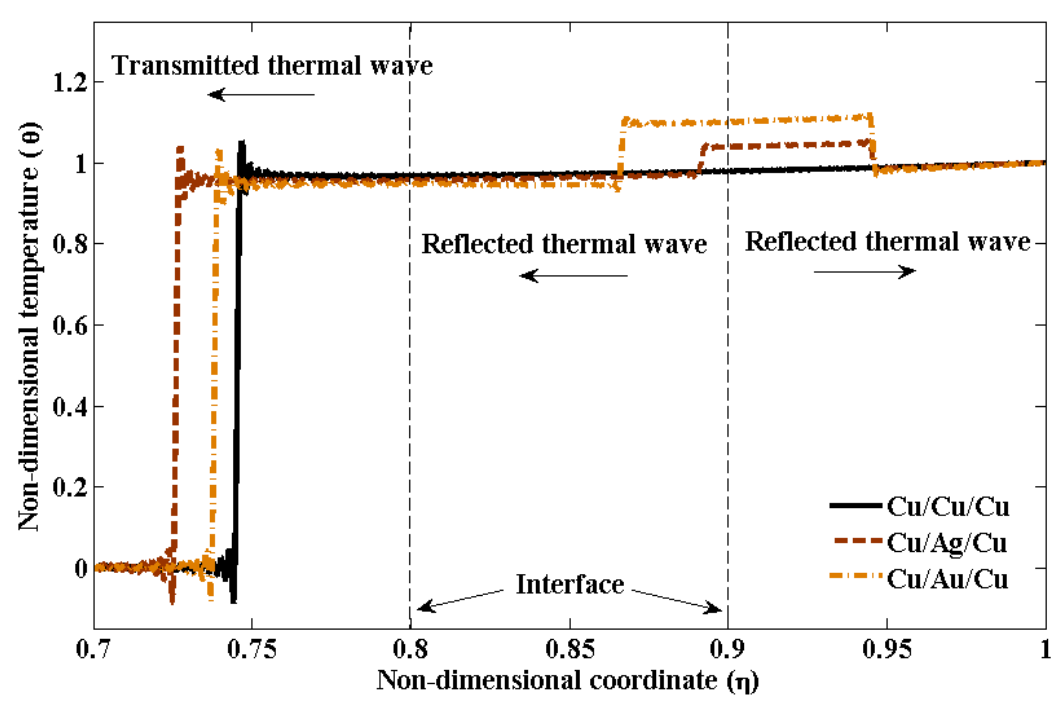

(b)

Fig. 10 Effect of material heterogeneity in the middle layer of a sandwich slab onto the temperature distribution at dimensionless time (a) $\zeta=0.084$ and (b) $\zeta=0.126$

$\left(m=0, n=3, \chi_{T}=0, \varepsilon_{0}=0.35, \delta_{0}=0.25, \alpha_{0}=0.15, C_{T}{ }^{2}=2\right.$, Hyperbolic TPL $)$

\subsection{Thermal Response of Sandwich Panels with Porous Cores}

We examine here sandwich panels with porous cores, commonly used for example in aerospace, electronics, and biomedicine. As expected, we show that when a thermal disturbance hits a sandwich panel, the relative density of the cellular core controls the thermal 
response. To do so, we examine a three-layer perfectly bonded sandwich cylinder $(m=1)$ with inner and outer solid layers of $C u$ and a porous middle layer of $C u$ with the relative density of $\rho_{r}$. The inner and outer radius of the sandwich cylinder are $x_{i}=0.7$ and $x_{o}=1$, respectively. The thermal compliance constants for all interfaces are expressed by the index: $\chi_{T}=\frac{\chi_{T}^{(j)} K^{(1)}}{x_{o}}(j=1,2, \ldots, N-1)$ and are assumed to experience no changes.

Figures 11 and 12 illustrate temperature and heat flux distribution obtained with the hyperbolic DPL model. The figures visualize the transient and steady-state temperature and heat flux distribution within the sandwich cylinder. The plots confirm the impact of relative density; in particular in porous layers, the thermal wave speed decreases with the relative density. Furthermore, a reduction of the relative density in the middle layer increases the transient temperature in the middle and outer layers of the cylinder, while it decreases the transient temperature in the inner layer. A decrease of the relative density also lessens the absolute value of the heat flux passing through the inner and middle layer, whereas it amplifies the thermal insulation of the inner surface from the thermal shock on the outer surface of the sandwich cylinder. Further investigations on the transient responses reveal that from one hand a reduced relative density of the middle layer decreases the steady-state temperature of the first half of the cylinder, far from the thermal excitation; from the other, it increases the steady-state temperature of the second half of the cylinder, close to the thermal disturbance. The heat flux within the cylinder, however, decreases with the relative density of the middle layer.

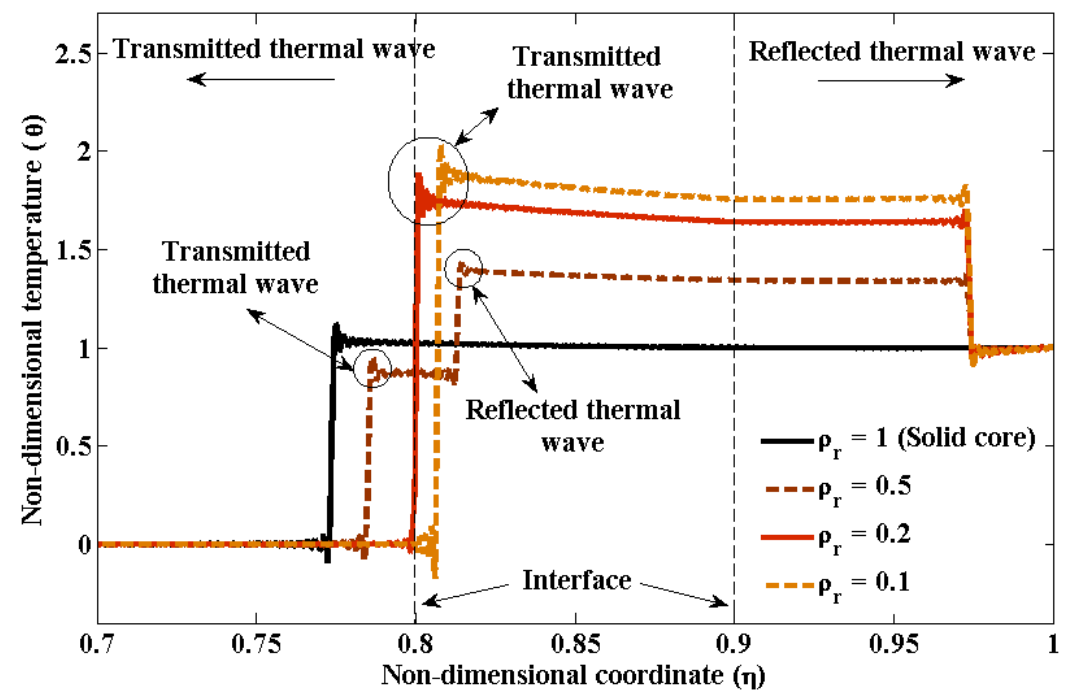

(a) 


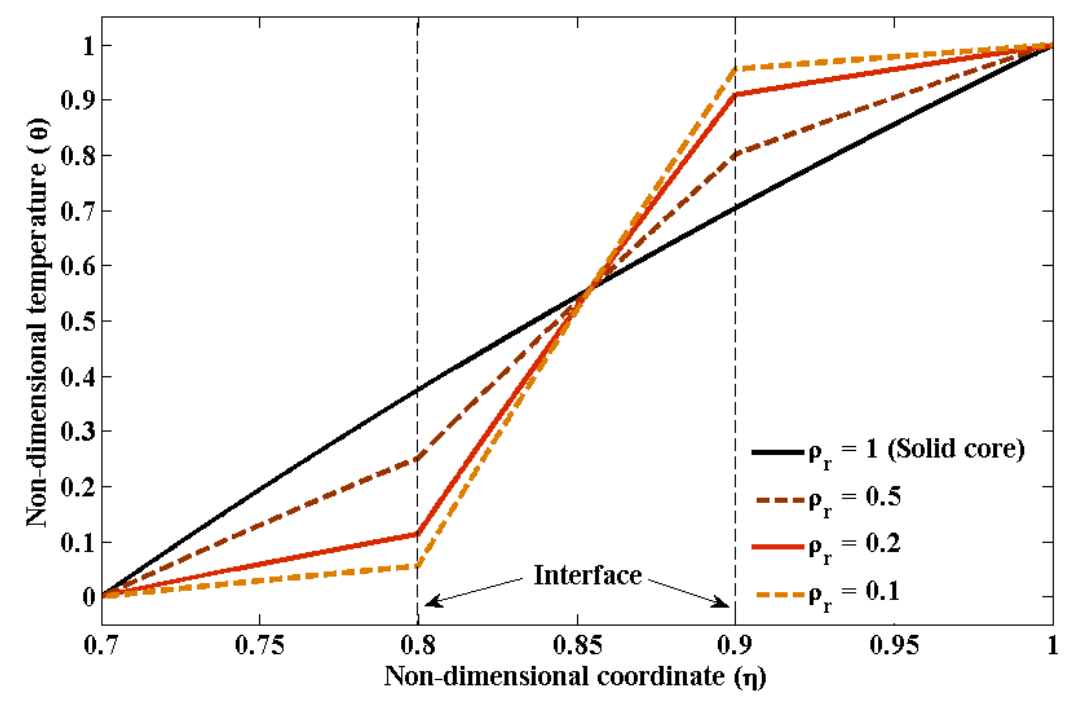

(b)

Fig. 11 Effect of relative density for the porous middle layer in a sandwich cylinder onto the temperature distribution at (a) dimensionless time $\zeta=0.112$ and (b) steady-state $\left(m=1, n=3, \chi_{T}=0, \varepsilon_{0}=0.35, \delta_{0}=0.25\right.$, Hyperbolic DPL $)$

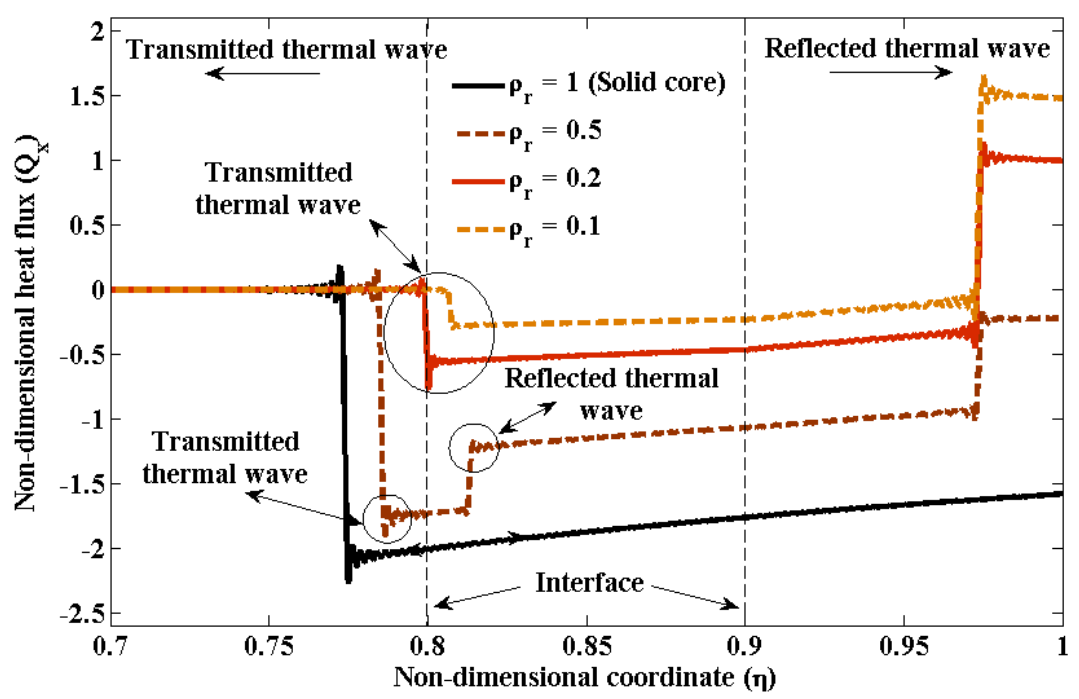

(a) 


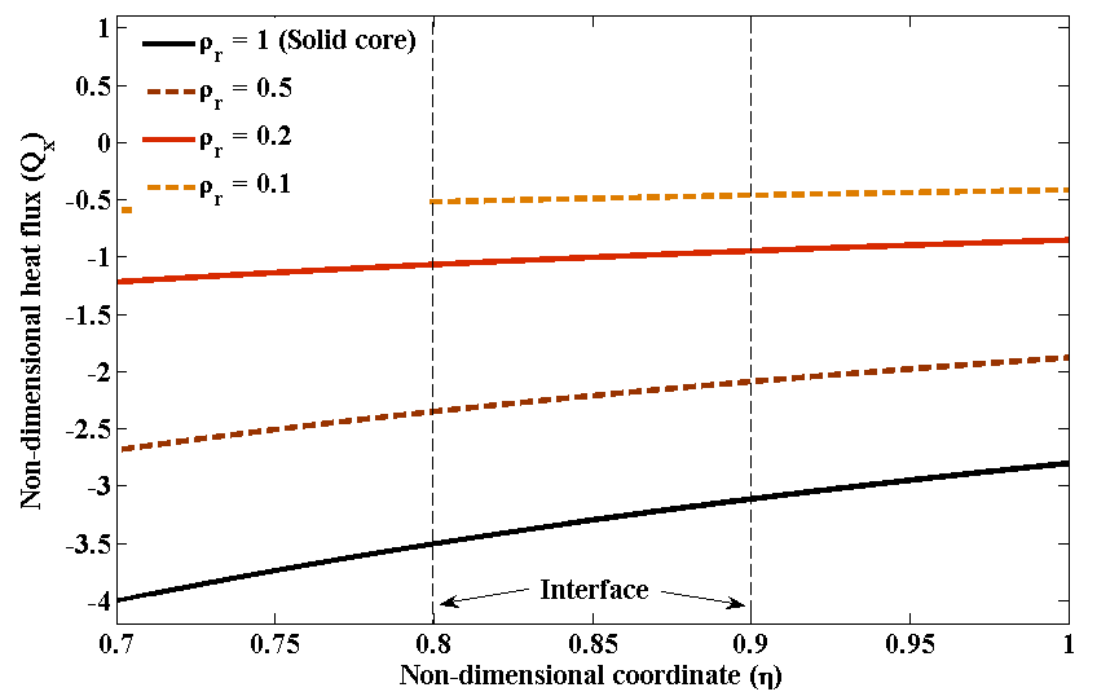

(b)

Fig. 12 Effect of relative density of the porous middle layer in a sandwich cylinder onto the heat flux distribution at (a) dimensionless time $\zeta=0.112$ and (b) steady-state

$$
\left(m=1, n=3, \chi_{T}=0, \varepsilon_{0}=0.35, \delta_{0}=0.25 \text {, Hyperbolic DPL }\right)
$$

\section{Concluding Remarks}

This paper has studied the heat conduction in 1D graded media with imperfect bonding interfaces through non-Fourier theories. A semi-analytic procedure combining Bessel functions and Laplace transform has been used to solve the governing differential equations of heat conduction through a medium with a finite thermal wave speed. We have shown there are differences in the thermal wave responses obtained with the hyperbolic heat conduction theories under investigation. In particular, we observed TPL predicts 5 percent temperature difference at the thermal wave front, higher than that obtained with DPL. The maximum transient temperature of the former is also $6 \%$ higher than the latter.

The application of the heat conduction analysis to FG solid and cellular materials has shown the critical role of the FGSM profile and the FGCM relative density. These material attributes have major control on the thermal response of the system. From one hand, we have observed the independence of the temperature time-history of the middle of FGC for all FGM profiles. From the other, we have appreciated the role of the FGM profile in governing the absolute value that the steady-state heat flux can reach. For E-FGM this value is higher than that for FORS-FGM and P-FGM.

The last part of the paper has examined the impact of bonding imperfection, bond heterogeneity, and the relative density of porous layers. As numerical results have shown, bonding imperfection and material heterogeneity are the main culprits for thermal wave separation; in this regard, we have captured the transmitted and reflected parts of the thermal wave propagating in a multilayered system. At the interface between imperfectly bonded 
layers, temperature has been observed to be discontinuous, as opposed to the heat flux. The thermal compliance of the interfaces increases the temperature difference between layers, while it decreases the heat flux transmitted through the interface. The relative density of the porous core in a sandwich panel has been shown to remarkably affect its thermal behavior. In particular, the thermal wave speed and the heat flux decrease with the relative density, whereas the transient temperature increases. This helps to gain insight into the importance of selecting the relative density that best optimizes the thermal management of a sandwich panel with porous core.

\section{Appendix A}

The non-zero components of $\left[K_{T P L}\right],\left\{X_{T P L}\right\}$, and $\left\{F_{T P L}\right\}_{2 N \times 1}$ in Eq. (16) are given as follows:

$$
\begin{gathered}
K_{T P L}(1,1)=\eta_{\gamma}^{\frac{1-m}{2}} J_{G^{(1)}}\left(I^{(1)} \eta_{\gamma}\right), K_{T P L}(1,2)=\eta_{\gamma}^{\frac{1-m}{2}} Y_{G^{(1)}}\left(I^{(1)} \eta_{\gamma}\right) \\
K_{T P L}(2 j, 2 j-1)=\frac{\chi_{T}^{(j)} K^{(1)}}{x_{o}} \frac{P_{T P L}^{(j)}}{2} \eta_{j+1}^{\frac{-m-1}{2}}\left(M^{(j)} J_{G^{(j)}}\left(I^{(j)} \eta_{j+1}\right)-2 I^{(j)} \eta_{j+1} J_{G^{(j)+1}}\left(I^{(j)} \eta_{j+1}\right)\right)-\eta_{j+1}^{\frac{1-m}{2}} J_{G^{(j)}}\left(I^{(j)} \eta_{j+1}\right) \\
K_{T P L}(2 j, 2 j)=\frac{\chi_{T}^{(j)} K^{(1)}}{x_{o}} \frac{P_{T P L}^{(j)}}{2} \eta_{j+1}^{\frac{-m-1}{2}}\left(M^{(j)} Y_{G^{(j)}}\left(I^{(j)} \eta_{j+1}\right)-2 I^{(j)} \eta_{j+1} Y_{G^{(j)}+1}\left(I^{(j)} \eta_{j+1}\right)\right)-\eta_{j+1} \frac{1-m}{2} Y_{G^{(j)}}\left(I^{(j)} \eta_{j+1}\right) \\
K_{T P L}(2 j, 2 j+1)=\eta_{j+1}^{\frac{1-m}{2}} J_{G^{(j+1)}}\left(I^{(j+1)} \eta_{j+1}\right), K_{T P L}(2 j, 2 j+2)=\eta_{j+1}{ }^{\frac{1-m}{2}} Y_{G^{(j+1)}}\left(I^{(j+1)} \eta_{j+1}\right) \\
K_{T P L}(2 j+1,2 j-1)=\frac{P_{T P L}^{(j)}}{2} \eta_{j+1}^{\frac{-m-1}{2}}\left(M^{(j)} J_{G^{(j)}}\left(I^{(j)} \eta_{j+1}\right)-2 I^{(j)} \eta_{j+1} J_{G^{(j)}+1}\left(I^{(j)} \eta_{j+1}\right)\right) \\
K_{T P L}(2 j+1,2 j)=\frac{P_{T P L}^{(j)}}{2} \eta_{j+1}^{\frac{-m-1}{2}}\left(M^{(j)} Y_{G^{(j)}}\left(I^{(j)} \eta_{j+1}\right)-2 I^{(j)} \eta_{j+1} Y_{G^{(j)}+1}\left(I^{(j)} \eta_{j+1}\right)\right) \\
K_{T P L}(2 j+1,2 j+1)=-\frac{P_{T P L}^{(j+1)}}{2} \eta_{j+1}^{\frac{-m-1}{2}}\left(M^{(j+1)} J_{G^{(j+1)}}\left(I^{(j+1)} \eta_{j+1}\right)-2 I^{(j+1)} \eta_{j+1} J_{G^{(j+1)}+1}\left(I^{(j+1)} \eta_{j+1}\right)\right) \\
K_{T P L}(2 j+1,2 j+2)=-\frac{P_{T P L}^{(j+1)}}{2} \eta_{j+1} \frac{-m-1}{2}\left(M^{(j+1)} Y_{G^{(j+1)}}\left(I^{(j+1)} \eta_{j+1}\right)-2 I^{(j+1)} \eta_{j+1} Y_{G^{(j+1)}+1}\left(I^{(j+1)} \eta_{j+1}\right)\right) \\
K_{T P L}(2 N, 2 N-1)=J_{G^{(N)}}\left(I^{(N)}\right), K_{T P L}(2 N, 2 N)=Y_{G^{(N)}}\left(I^{(N)}\right) \\
(j=1,2, \ldots, N-1)(\mathrm{A} .1)
\end{gathered}
$$

and

$$
F_{T P L}(1,1)=\theta_{w i} \tilde{f}_{i}(s), F_{T P L}(2 N, 1)=\theta_{w o} \tilde{f}_{o}(s)
$$

\section{Appendix B}


Herein, the non-zero components of $\left[K_{s}\right],\left\{X_{s}\right\}$, and $\left\{F_{s}\right\}$ in Eq. (16) are specified. For Cartesian $(m=0)$ and spherical $(m=2)$ coordinate, we have:

$$
\begin{gathered}
K_{s}(1,1)=1, K_{s}(1,2)=\eta_{\gamma}{ }^{1-m} \\
K_{s}(2 j, 2 j-1)=-1, K_{s}(2 j, 2 j)=-\frac{\chi_{T}^{(j)} K^{(j)}}{x_{o}}(1-m) \eta_{j+1}^{-m}-\eta_{j+1}^{1-m} \\
K_{s}(2 j, 2 j+1)=1, K_{s}(2 j, 2 j+2)=\eta_{j+1}^{1-m} \\
K_{s}(2 j, 2 j)=-\frac{K^{(j)}}{K^{(1)}}(1-m) \eta_{j+1}^{-m}, K_{s}(2 j, 2 j+2)=-\frac{K^{(j+1)}}{K^{(1)}}(1-m) \eta_{j+1}^{-m} \\
K_{s}(2 N, 2 N-1)=1, K_{s}(2 N, 2 N)=1 \quad(j=1,2, \ldots, N-1)
\end{gathered}
$$

and

$$
F_{s}(1,1)=\theta_{w i}, F_{s}(2 N, 1)=\theta_{w o}
$$

For cylindrical coordinate $(m=1)$, we have:

$$
\begin{gathered}
K_{s}(1,1)=1, K_{s}(1,2)=\ln \left(\eta_{\gamma}\right) \\
K_{s}(2 j, 2 j)=-\frac{K^{(j)}}{K^{(1)}} \frac{1}{\eta_{i+1}}, K_{s}(2 j, 2 j+2)=\frac{K^{(j+1)}}{K^{(1)}} \frac{1}{\eta_{j+1}} \\
K_{s}(2 j+1,2 j-1)=-1, K_{s}(2 j+1,2 j)=-\frac{\chi_{T}^{(j)} K^{(j)}}{x_{o}} \frac{1}{\eta_{j+1}}-\ln \left(\eta_{j+1}\right) \\
K_{s}(2 j+1,2 j+1)=1, K_{s}(2 j+1,2 j+2)=\ln \left(\eta_{j+1}\right) \\
K_{s}(2 N, 2 N-1)=1 \quad(j=1,2, \ldots, N-1)
\end{gathered}
$$

and

$$
F_{s}(1,1)=\theta_{w i}, F_{s}(2 N, 1)=\theta_{w o}
$$

\section{References:}

[1] A.H. Akbarzadeh, Z.T. Chen, Transient heat conduction in a functionally graded cylindrical panel based on the dual phase lag theory, Int. J. Thermophys. 33 (2012) 11001125 .

[2] D. Chandrasekharaiah, Hyperbolic thermoelasticity: a review of recent literature, Appl. Mech. Rev. 51 (12) (1998) 705-729.

[3] D.Y. Tzou, The generalized lagging response in small-scale and high-rate heating, Int. J. Heat Mass Transfer 38 (17) (1995) 3231-3240.

[4] D. Tzou, A unified field approach for heat conduction from macro-to micro-scales, J. Heat Trans. 117 (1) (1995) 8-16. 
[5] S.K.R. Choudhuri, On a thermoelastic three-phase-lag model, J. Therm. Stresses, 30 (3) (2007) 231-238.

[6] M.A. Ezzat, A.A. El-Bary, M.A. Fayiki, Fractional Fourier law with three-phase-lag of thermoelasticity, Mech. Adv. Mater. Struct. 20 (2013) 593-602.

[7] M.A. Ezzat, A.S. El Karamany, M.A. Fayik, Fractional order theory in thermoelastic solid with three-phase lag heat transfer, Arch. Appl. Mech. 82 (2012) 557-572.

[8] D.W. Tang, N. Araki, Non-Fourier heat conduction in a finite medium under periodic surface thermal disturbance, Int. J. Heat Mass Trans. 39 (8) (1996) 1585-1590.

[9] A.H. Bishri, Modelling non-Fourier heat conduction with periodic thermal oscillation using the finite integral transform, Appl. Math. Model. 23 (1999) 899-914.

[10] N.M. Abdel-Jabbar, M.A. Al-Nimr, The dual-phase-lag heat conduction model in thin slab under fluctuating thermal disturbance, Heat Transfer Eng. 24 (5) (2003) 47-54.

[11] M. Xu, L. Wang, Dual-phase-lagging heat conduction based on Boltzmann transport equation, Int. J. Heat Mass Transfer 48 (2005) 5616-5624.

[12] K.C. Liu, P.C. Chang, Analysis of dual-phase-lag heat conduction in cylindrical system with a hybrid method, Appl. Math. Model. 31 (2007) 369-380.

[13] M.H. Babaei, Z.T. Chen, Hyperbolic heat conduction in a functionally graded hollow sphere, Int. J. Thermophys. 29 (2008) 1457-1469.

[14] K. Ramadan, Semi-analytical solutions for the dual-phase-lag heat conduction in multilayered media, Int. J. Therm. Sci. 48 (1) (2009) 14-25.

[15] A. Miranville, R. Quintanilla, A phase-field model based on a three-phase-lag eat conduction, Appl. Math. Optim, 63 (2011) 133-150.

[16] H.D. Wang, B.Y. Cao, Z.Y. Guo, Non-Fourier heat conduction in carbon nanotubes, J. Heat Transfer, 134 (2012) 051004-1-6.

[17] M. Wang, N. Yang, Z.Y. Guo, Non-Fourier heat conduction in nanomaterials, J. Appl. Phys., 110 (2011) 064310-1-7.

[18] K.Q. Hu, Z.T. Chen, Transient heat conduction analysis of a cracked half-plane using dual-phase-lag theory, Int. J. Heat Mass Trans. 62 (2013) 445-451.

[19] N. Afrin, Y. Zhang, J.K. Chen, Dual-phase lag behavior of a gas-saturated porousmedium heated by a short-pulsed laser, Int. J. Therm. Sci. 75 (2014) 21-27.

[20] M.H. Babaei, Z.T. Chen, The transient coupled thermo-piezoelectric response of a functionally graded piezoelectric hollow cylinder to dynamic loading, Proc. R. Soc. A 466 (2010) 1077-1091.

[21] A.H. Akbarzadeh, M.H. Babaei, Z.T. Chen, Coupled thermopiezoelectric behaviour o a one-dimensional functionally graded piezoelectric medium based on $\mathrm{C}-\mathrm{T}$ theory, Proc. IMechE Part C: J. Mech. Eng. Sci. 225 (11) (2011) 2537-2551. 
[22] A.H. Akbarzadeh, M.H. Babaei, Z.T. Chen, Thermopiezoelectric analysis of a functionally graded piezoelectric medium, Int. J. Appl. Mech. 3 (1) (2011) 47-68.

[23] S.K. Hosseini zad, A. Komeili, M.R. Eslami, S. Fariborz, Classical and generalized coupled thermoelasticity analysis in one-dimensional layered media, Arch. Appl. Mech. 82 (2012) 267-282.

[24] S. Banik, M. Kanoria, Generalized thermoelastic interaction in a functionally graded isotropic unbounded medium due to varying heat source with three-phase-lag effect, Math. Mech. Solids 18 (3) (2012) 231-245.

[25] A.H. Akbarzadeh, M. Abbasi, M.R. Eslami, Coupled thermoelasticity of functionally graded plates based on the third-order shear deformation theory, Thin-Walled Struct. 53 (2012) 141-155.

[26] A.H. Akbarzadeh, M. Abbasi, S.K. Hosseini zad, M.R. Eslami, Dynamic analysis of functionally graded plates using the hybrid Fourier-Laplace transform under thermomechanical loading, Meccanica 46 (2011) 1373-1392.

[27] A.H. Akbarzadeh, D. Pasini, Multiphysics multilayered and functionally graded cylinders under prescribed hygro-thermo-magnetoc-electro-mechanical loading, ASME J. Appl. Mech. (2013) DOI:10.1115/1.4025529.

[28] C.F. Tsai, G. Nixon, Transient temperature distribution of a multilayer composite wall with effects of internal thermal radiation and conduction, Num. Heat Transfer 10 (1986) 95101.

[29] A.K. Noor, W.S. Burton, Steady-state heat conduction in multilayered composite plates and shells, Comput. Struct. 39 (1-2) (1991) 185-193.

[30] A.L. Kalamkarov, B.A. Kudryavtsev, and O.B. Rudakova, Heat conduction in a multilayer composite wedge, J. Eng. Phys. Thermophys. 64 (4) (1993) 396-400.

[31] J. Argyris, L. Tenek, F. Oberg, A multilayer composite triangular element for steadystate conduction/convection/radiation het transfer in complex shells, Comput. Meth. Appl. Mech. Eng. 120 (1995) 271-301.

[32] K.A. Anotonopoulos, C. Tzivnidis, Analytical solution of boundary value problems of heat conduction in composite regions with arbitrary convection boundary conditions, Acta Mech. 118 (1996) 65-78.

[33] J.Q. Tarn, Y.M. Wang, End effects of heat conduction in circular cylinders of functionally graded materials and laminated composites, Int. J. Heat Mass Transfer 47 (2004) 5741-5747.

[34] Y. Sun, I.S. Wichman, On transient heat conduction in a one-dimensional composite slab, Int. J. Heat Mass Transfer 47 (2004) 1555-1559. 
[35] X. Lu, P. Tervola, M. Viljanen, An efficient analytical solution to transient heat conduction in a one-dimensional hollow composite cylinder, J. Phys. A: Math. Gen. 38 (2005) 10145-10155.

[36] B. Yang, H. Shi, A thermal stability criterion for heat conduction in multilayer composite solids, J. Heat Transfer 131 (11) (2009) 111304-1-7.

[37] P.K. Jain, S. Singh, R. Uddin, Analytical solution to transient asymmetric heat conduction in a multilayer annulus, J. Heat Transfer 131 (1) (2009) 011304-1-7.

[38] A. Amiri Delouei, M.H. Kayhani, M. Norouzi, Exact analytical solution of unsteady axisymmetric conductive heat transfer in cylindrical orthotropic composite laminates, Int. J. Heat Mass Transfer 55 (2012) 4427-4436.

[39] A.H. Akbarzadeh, Z.T. Chen, Hygrothermal stresses in one-dimensional functionally graded piezoelectric media in constant magnetic field, Compos. Struct. 97 (2013) 317-331.

[40] A.H. Akbarzadeh, Z.T. Chen, Magnetoelectroelastic behavior of rotating cylinders resting on an elastic foundation under hygrothermal loading, Smart Mater. Struct. 21 (2) (2012) 125013 (17pp).

[41] M.V. Timoshenko, Numerical simulation of heat transfer in multilayer structures with generalized nonideal contact, J. Eng. Phys. Thermophys. 69 (5) (1996) 590-595.

[42] I. Lekakis, M.A. Kattis, E. Providas, A.L. Kalamkarov, The disturbance of heat flow and thermal stresses in composites with partially bonded inclusions, Compos. Part B 31 (2000) 21-27.

[43] Z.Q. Cheng, R.C. Batra, Thermal effects on laminated composite shells containing interfacial imperfections, Compos. Struct. 52 (2001) 3-11.

[44] Y. Liu, A. Mioduchowski, C.Q. Ru, Effect of imperfect interface on thermal stressesassisted matrix cracking in fiber composites, J. Therm. Stresses 25 (2002) 585-599.

[45] D. Duschlbauer, H.E. Pettermann, H.J. Bohm, Heat conduction of spheroidal inhomogeneity with imperfectly bonded interface, J. Appl. Phys. 94 (3) (2003) 1539-1549.

[46] H.L. Duan, B.L. Karihaloo, Effective thermal conductivities of heterogeneous media containing multiple imperfectly bonded inclusions, Phys. Rev. B 75 (2007) 064206-1-9.

[47] H. Hatami-Marbini, H.M. Shodja, On thermoelastic fields of multi-phase inhomogeneity system with perfectly/imperfectly bonded interfaces, Int. J. Solids Struct. 45 (2008) 58315843.

[48] J.N. Reddy, C.D. Chin, Thermomechanical analysis of functionally graded cylinders and plates, J. Therm. Stresses 21 (1998) 593-626.

[49] V. Birman, L.W. Byrd, Modeling and analysis of functionally graded materials and structures, Appl. Mech. Rev. 60 (2007) 195-216. 
[50] A.H. Akbarzadeh, M.H. Babaei, Z.T. Chen, The thermo-electromagnetoelastic behavior of a rotating functionally graded piezoelectric cylinder, Smart Mater. Struct. 20 (6) (2011) 065008.

[51] A. Sutradhar, G.H. Paulino, L.J. Gray, Transient heat conduction in homogenous and non-homogenous materials by the Laplace transform Galerkin boundary element method, Eng. Analysis Bound Elem. 26 (2002) 119-132.

[52] J. Sladek, V. Sladek, C. Zhang, Transient heat conduction analysis in functionally graded materials by the meshless local boundary integral equation method, Comput. Mater. Sci. 28 (2003) 494-504.

[53] B. Chen, L. Tong, Y. Gu, H. Zhang, O. Ochoa, Transient heat transfer analysis of functionally graded materials using adaptive precise time integration and graded finite elements, Numer. Hear Transfer, Part B 45 (2004) 181-200.

[54] L.F. Qian, R.C. Batra, Three-dimensional transient heat conduction in a functionally graded thick plate with a higher-order plate theory and a meshless local Petrov-Galerkin method, Comput. Mech. 35 (2005) 214-226.

[55] H. Wang, Q.H. Qin, Y.L. Kang, A meshless model for transient heat conduction in functionally graded materials, Comput. Mech. 38 (2006) 51-60.

[56] S.M. Hosseini, M. Akhlaghi, M. Shakeri, Transient heat conduction in functionally graded thick hollow cylinders by analytical method, Heat Mass Transfer 43 (2007) 669-675.

[57] A. Khosravifard, M.R. Hematiyan, L. Marin, Nonlinear heat conduction analysis of functionally graded materials in the presence of heat sources using an improved meshless radial point interpolation method. Appl. Math. Model. 35 (2011) 4157-4174.

[58] T. Darabseh, N. Yilmaz, M. Bataineh, Transient thermoelasticity analysis of functionally graded thick hollow cylinder based on Green-Lindsay model, Int. J. Mech. Mater. Des. 8 (2012) 247-255.

[59] L.L. Cao, Q.H. Qin, N. Zhao, Hybrid graded element model for transient heat conduction in functionally graded materials, Acta Mech. Sin. 28 (1) (2012) 128-139.

[60] T. Ishiguro, A. Makino, N. Araki, N. Noda, Transient temperature response in functionally gradient materials, Int. J. Thermophys. 14 (1993) 101-121.

[61] Y. Tanigawa, T. Akai, R. Kawamura, N. Oka, Transient heat conduction and thermal stress problems of a nonhomogeneous plate with temperature-dependent material properties, J. Therm. Stresses, 19 (1996) 77-102.

[62] Z.H. Jin, An asymptotic solution of temperature field in a strip of a functionally graded material, Int. Comm. Heat Mass Transfer 29 (7) (2002) 887-895.

[63] Y.T. Zhou, K.Y. Lee, D.H. Yu, Transient heat conduction in a functionally graded strip in contact with well stirred fluid with an outside heat source, Int. J. Heat Mass Trans. 54 (2011) 5438-5443. 
[64] Y.C. Yang, W.L. Chen, H.M. Chou, J.L.L. Salazar, Inverse hyperbolic thermoelastic analysis of a functionally graded hollow circular cylinder in estimating surface heat flux and thermal stresses, Int. J. Heat Mass Trans. 60 (2013) 125-133.

[65] H.M. Wang, An effective approach for transient thermal analysis in a functionally graded hollow cylinder, Int. J. Heat Mass Trans. 67 (2013) 499-505.

[66] M.F. Ashby, A.G. Evans, N.A. Fleck, L.J. Gibson, J.W. Hutchinson, H.N.G. Wadley, Metal Foams: A Design Guide, Butterworth-Heinemann, Boston, 2000.

[67] M.F. Ashby, Materials selection in mechanical design, Elsevier Ltd. (2011).

[68] K. Boomsma, D. Poulikakos, On the effective thermal conductivity of a threedimensionally structured fluid-saturated metal foam, Int. J. Heat Mass Trans. 44 (2001) 827836.

[69] A. Bhattacharya, V.V. Clamidi, R.L. Mahajan, Thermophysical properties of high porosity metal foams, Int. J. Heat Mass Trans. 45 (2002) 1017-1031.

[70] S. Venkataraman, M.L. Blosser, Optimal functionally graded metallic foam thermal insulation. AIAA J. 42 (11) (2004) 2355-2363.

[71] D.A. Nield, A. Bejan, Convection in Porous Media, Springer, New York, 2006.

[72] K.C. Leong, H.Y. Li, Theoretical study of the effective thermal conductivity of graphite foam based on a unit cell model, Int. J. Heat Mass Trans. 54 (2011) 5491-5496.

[73] E. Sadeghi, S. Hsieh, M. Bahrami, Thermal conductivity and contact resistance of metal foams, J. Phys. D: Appl. Phys. 44 (2011) 125406(7pp).

[74] K.S. Challagulla, T.A. Venkatesh, Electromechanical response of piezoelectric foams. Acta Mater. 60 (2012) 2111-2127.

[75] N. Gupta, A functionally graded syntatic foam material for high energy absorption under compression, Mater. Lett. 61 (2007) 979-982.

[76] C. Zhou, P. Wang, W. Li, Fabrication of functionally graded porous polymer via supercritical $\mathrm{CO}_{2}$ foaming, Compos. Part B 42 (2011) 318-325.

[77] H. Zhu, B.V. Sankar, R.T. Haftka, S. Venkataraman, M. Blosser, Optimization of functionally graded metallic foam insulation under transient heat transfer conditions, Struct. Multidisc. Optim. 28 (2004) 349-355.

[78] A.H. Akbarzadeh, Z.T. Chen, Heat conduction in one-dimensional functionally graded media based on the dual-phase-lag theory, Proc. IMechE Part C: J. Mech. Eng. Sci. 227 (4) (2012) 744-759.

[79] A.H. Akbarzadeh, J.W. Fu, Z.T. Chen, Three-phase-lag heat conduction in a functionally graded hollow cylinder, Trans. Can. Soc. Mech. Eng. 37 (4) (2013) (In Press).

[80] R. Quintanila, R. Racke, Qualitative aspects in dual-phase-lag heat conduction, Proc. R. Soc. A 463 (2007) 659-674. 
[81] T. Chen, Thermal conduction of a circular inclusion with variable interface parameter, Int. J. Solids Struct. 38 (2001) 3081-3097.

[82] Z.Q. Cheng, A.K. Jemah, F.W. Williams, Theory of multilayered anisotropic plates with weakened interfaces, ASME J. Appl. Mech. 63 (1996) 1019-1026.

[83] A.H. Akbarzadeh, Z.T. Chen, On the harmonic magnetoelastic behavior of a composite cylinder with an embedded polynomial eigenstrain, Compos. Struct. 106 (2013) 296-305.

[84] F. Durbin, Numerical inversion of Laplace transforms: an efficient improvement to Dubner and Abate's method, Comput. J. 17 (4) (1974) 371-376.

[85] Z.T. Chen, A.H. Akbarzadeh, M.H. Babaei, Thermopiezoelectric response of a onedimensional functionally graded piezoelectric medium to a moving heat source-a review, Appl. Mech. Mater., 151 (2012) 396-400.

[86] R. Chein, H. Yang, T.H. Tsai, C. Lu, Experimental study of heat sink performance using copper foams fabricated by electroforming, Microsyst Technol 16 (2010) 1157-1164.

[87] P.J. Antaki, Key features of analytical solutions for hyperbolic heat conduction, $30^{\text {th }}$ AIAA Thermophys. Confererence, San Diego CA, 95-2044 (1995), 1-15.

[88] Y. Zhang, Generalized dual-phase lag bioheat equations based on nonequilibrium heat transfer in living biological tissues, Int. J. Heat Mass Trans. 52 (2009) 4829-4834.

[89] L. Zhou, J. Lin, H. Lin, G. Chen, Electrical-thermal switching effect in high-density polyethylene/graphite nanosheets conducting composites, J. Mater. Sci. 43 (2008) 4886-4891.

[90] A.H. Akbarzadeh, Z.T. Chen, Dual phase lag heat conduction in functionally graded hollow spheres, Int. J. Appl. Mech. 6 (1) (2014) 450002 (27 pages) DOI: 10.1142/S1758825114500021.

[91] M. Jaunicha, S. Rajea, K. Kimb, K. Mitraa, and Z. Guo, Bio-heat transfer analysis during short pulse laser irradiation of tissues, Int. J. Heat Mass Trans. 51 (2008) 5511-5521.

[92] F. Jiang, D. Liu, J. Zhou, Non-Fourier heat conduction phenomena in porous material heated by microsecond porous material heated by microsecond laser pulse, Microscale Therm. Eng., 6 (2002) 331-346.

[93] M. Wang, N. Pan, Modeling and prediction of the effective thermal conductivity of random open-cell porous foams, Int. J. Heat Mass Trans. 51 (2008) 1325-1331.

[94] M. Elsayed, D. Pasini, Analysis of the Elastostatic Specific Stiffness of 2D StretchingDominated Lattice Materials, J. Mech. Mater., 42 (7) (2010), 709-725.

[95] A. Vigliotti, D. Pasini, Stiffness and Strength of Tridimensional Periodic Lattices, Compu. Meth. Appl. Mech. Eng., 229-232 (2012) 27-43.

[96] A. Vigliotti, D. Pasini, Linear multiscale analysis and finite element validation of stretching and bending dominated lattice materials, J. Mech. Mater., 46 (2012), 57-68. 
[97] S.A. Khanoki, D. Pasini, Mechanical properties of planar lattice materials via asymptotic homogenization and comparison with alternative homogenization methods, Int. J. Mech. Sci. 77 (2013) 249-262.

[98] A.H. Akbarzadeh, Z.T. Chen, Magnetoelastic Field of a Multi-Layered and Functionally Graded Cylinder with a Dynamic Polynomial Eigenstrain, ASME J. Appl. Mech. 81 (2) (2013) 021009.

[99] A.H. Akbarzadeh, S.K. Hosseini zad, M. Abbasi, M.R. Eslami, Mechanical behavior of functionally plates under static and dynamic loading, IMechE Part C: J. Mech. Eng. Sci. 225 (2) (2011) 326-333.

[100] S.H. Chi, Y.L. Chung, Mechanical behavior of functionally graded material plates under transverse load-Part I: Analysis, Int. J. Solids Struct. 43 (2006) 3657-3674.

[101] A. Komeili, A.H. Akbarzadeh, A. Doroushi, M.R. Eslami, Static Analysis of Functionally Graded Piezoelectric Beams under Thermo-Electro-Mechanical Load, Adv. Mech. Eng. 2011 (2011) 10 pages, DOI:10.1155/2011/153731.

[102] Y. Kiani, A.H. Akbarzadeh, Z.T. Chen, M.R. Eslami, Static and dynamic analysis of an FGM doubly curved panel resting on the Pasternak-type elastic foundation, Compos. Struct. 94 (8) (2012) 2474-2484. 